\title{
RADIATION CONDITIONS AT THE TOP OF A ROTATIONAL CUSP IN THE THEORY OF WATER-WAVES *
}

\author{
SERGEY A. NAZAROV ${ }^{1}$ AND JARI TASKINEN ${ }^{2}$
}

\begin{abstract}
We study the linearized water-wave problem in a bounded domain (e.g. a finite pond of water) of $\mathbb{R}^{3}$, having a cuspidal boundary irregularity created by a submerged body. In earlier publications the authors discovered that in this situation the spectrum of the problem may contain a continuous component in spite of the boundedness of the domain. Here, we proceed to impose and study radiation conditions at a point $\mathcal{O}$ of the water surface, where a submerged body touches the surface (see Fig. 1). The radiation conditions emerge from the requirement that the linear operator associated to the problem be Fredholm of index zero in relevant weighted function spaces with separated asymptotics. The classification of incoming and outgoing (seen from $\mathcal{O}$ ) waves and the unitary scattering matrix are introduced.
\end{abstract}

Mathematics Subject Classification. 76B15, 35J25, 35P99.

Received January 11, 2010. Revised September 30, 2010.

Published online May 27, 2011

\section{INTRODUCTION}

\subsection{Preamble}

We consider the linearized water-wave problem in a bounded domain $\Omega$, like a finite volume water pond containing a submerged body, touching the water surface in a single point $\mathcal{O}$, see Figures 1 and 2. The problem consists of the Poisson equation in $\Omega$ for the unknown $\varphi$ and given $f$,

$$
-\Delta_{x} \varphi(x)=f(x), \quad x \in \Omega,
$$

homogeneous Neumann conditions $\partial_{\nu} \varphi(x)=0\left(\partial_{\nu}\right.$ is the normal derivative) on the boundary except for the water surface $\Gamma$, where a Steklov type spectral condition $\partial_{\nu} \varphi(x)=\lambda \varphi(x)$ is posed with $\lambda \in \mathbb{C}$ as a spectral parameter. At $\mathcal{O}$ the two tangential boundary components create a thinning water blanket, which is cuspidal in the vertical direction. This geometry makes the asymptotic behaviour of the solutions $\varphi$ as $x \rightarrow \mathcal{O}$ quite complicated. We shall consider the above problem in the weak formulation, which transforms it into a more standard spectral problem of an unbounded operator in a Hilbert space. As a consequence of the geometry,

\footnotetext{
Keywords and phrases. Linear water-wave problem, cuspidal domain, radiation condition, scattering matrix.

* The first named author was supported by the RFFI, grant 09-01-00759 and by the Academy of Finland, grant 139545. The second named author was supported by the Academy of Finland project 134234.

1 Institute of Mechanical Engineering Problems, V.O., Bolshoi pr., 61, 199178, St. Petersburg, Russia.

${ }^{2}$ University of Helsinki, Department of Mathematics and Statistics, P.O. Box 68, 00014 Helsinki, Finland.

taskinen@cc.helsenki.fi
} 


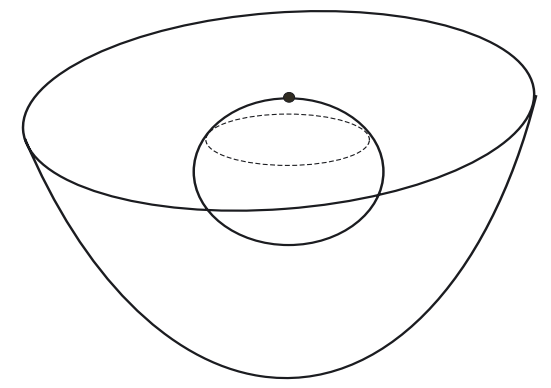

Figure 1. Domain $\Omega$ with rotational cusp.

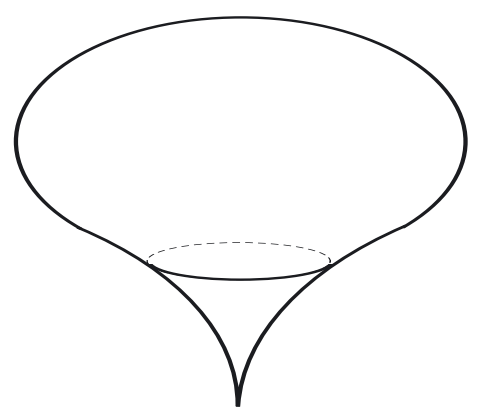

Figure 3. Domain with peak.

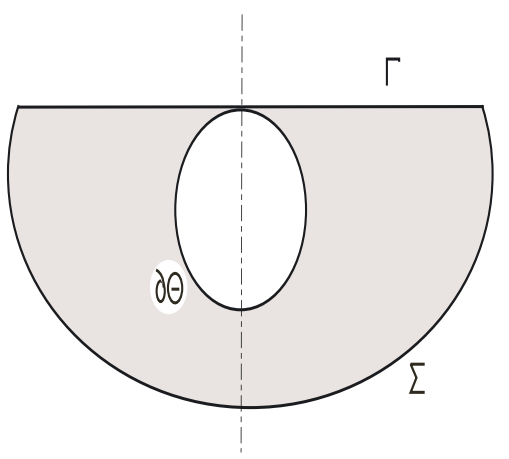

Figure 2. Cross-section of $\Omega$.

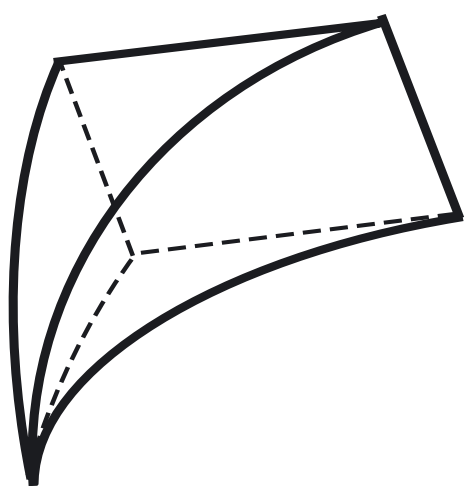

FiguRE 4. Domain with beak.

the problem is ill-posed in the Sobolev space $H^{1}(\Omega)$. Notice that in presented cuspidal domains the embedding $H^{1}(\Omega) \subset L^{2}(\Gamma)$ may lose compactness or even fail, see $[7,8,18,22]$. In fact it is shown in [38] that the essential spectrum is nonempty: it contains a continuous component.

However, finding the essential spectrum is not sufficient to make the boundary value problem well-posed. The main goal of the present paper becomes to provide a correct formulation of the above linear water-wave problem by introducing radiation conditions at the point $\mathcal{O}$. To that end, we shall study the Fredholm properties of the associated linear operator $A_{\beta}(\lambda)$ in appropriate weighted function spaces, the weights related to the asymptotic behaviour of the solutions around $\mathcal{O}$. The radiation conditions emerge from the requirement that $A_{\beta}(\lambda)$ be Fredholm of index zero in a carefully chosen function space.

There exist several methods to impose appropriate radiation conditions in unbounded domains, but they are closely connected with the geometry of the outlets to infinity, and therefore cannot directly be applied for bounded domains.

Our results show that cuspidal irregularity may act as a "black hole" for water waves: incoming waves may propagate an infinite time towards the cusp. For elastic and acoustic waves in finite volume bodies, black holes are related to the appearance of the continuous spectrum in cuspidal solids, like in Figures 3 and 4 with peakand beak-shaped solids. The elasticity system in irregular domains has been studied in the papers $[1,6,30,32]$. Notice that black holes have also been experimentally verified for elastic peaks by Mironov [23]. It is remarkable that cuspidal irregularities are nowadays used in specific engineering devices, like wave filters and dampers (see, e.g. $[5,14]$ and again [23]). At the end of our paper we continue the discussion on the physical nature of the radiation conditions. 
We mention that in the more simple case of the Steklov spectral problem for Laplace operator (i.e. the spectral boundary condition prevailing all around the boundary instead of the homogeneous Neumann condition) the existence of continuous spectrum in peak-shaped domain was shown in [31,37].

\subsection{Formulation of the water wave problem in weighted function spaces}

In this section we first present the detailed assumptions on the problem under consideration, its weak formulation and definitions of the appropriate function spaces, and finally consider the linear operator $A_{\beta}(\lambda)$ associated to the problem. The domain $\Omega$ of the lower half-space $\mathbb{R}_{-}^{3}$ has compact closure $\bar{\Omega}=\Omega \cup \partial \Omega$ and its boundary $\partial \Omega$ consists of three parts (see Fig. 2), namely the horizontal water surface

$$
\Gamma=\left\{x=(y, z): z=x_{3}=0, y=\left(x_{1}, x_{2}\right) \in \Gamma^{\prime}\right\} \subset \partial \mathbb{R}_{-}^{3},
$$

the wall and bottom $\Sigma \subset \mathbb{R}_{-}^{3}$ of the pond, and the surface $\partial \Theta$ of the submerged body $\Theta$. We assume $\partial \Omega$ to be Lipschitz everywhere except at the origin $\mathcal{O}$ of the Cartesian coordinate system $x=\left(x_{1}, x_{2}, x_{3}\right)$. Furthermore, in a neighbourhood $\mathcal{U}$ of $\mathcal{O}$ the domain $\Omega$ is given by the relation

$$
-h(y)<z<0,
$$

where $h$ is a smooth function in the variables $y=\left(y_{1}, y_{2}\right)$ such that

$$
\begin{array}{r}
h(0)=0, \nabla_{y} h(0)=0, h(y)=H(y)+\mathcal{O}\left(|y|^{3}\right), y \rightarrow 0, \\
H(y)=H_{11} y_{1}^{2}+2 H_{12} y_{1} y_{2}+H_{22} y_{2}^{2}>0 \text { for } y \neq 0 .
\end{array}
$$

In other words, the surface $\partial \Theta$ touches the plane $\partial \mathbb{R}_{-}^{3} \supset \Gamma$ at $\mathcal{O}$.

Also the following notation will be used. Polar coordinates for the variable $y=\left(y_{1}, y_{2}\right) \in \partial \mathbb{R}_{-}^{3}$ are denoted by $(r, \vartheta)$, where $r=|y|$ and $\vartheta$ is the angular variable on the circle $\mathbb{S}^{1}$. Punctured sets are denoted by $\partial \Omega_{\bullet}=\partial \Omega \backslash \mathcal{O}$, $\Gamma_{\bullet}=\Gamma \backslash \mathcal{O}, \partial \Theta \bullet=\partial \Theta \backslash \mathcal{O}$, and $\mathbb{R}_{\bullet}^{2}=\mathbb{R}^{2} \backslash \mathcal{O}$. The water blanket $\Xi_{R}$ is the subdomain

$$
\Xi_{R}:=\left\{(y, z) ; y \in \mathbb{B}_{R}, 0>z>-h(y)\right\},
$$

where $\mathbb{B}_{R}=\{y: r<R\}$ is a disk of radius $R>0$ and $R$ is so small that $\Xi_{R} \subset \mathcal{U}$; it will be necessary later to choose $R$ small enough.

We consider the boundary value problem of linearized water-wave theory (see, e.g., $[15,41]$ )

$$
\begin{aligned}
& -\Delta_{x} \varphi(x)=f(x), \quad x \in \Omega, \\
& \partial_{\nu} \varphi(x)=0, \quad x \in \Sigma \cup \partial \Theta_{\bullet}, \\
& \partial_{z} \varphi(x)=\lambda \varphi(x), \quad x \in \Gamma_{\text {. }} .
\end{aligned}
$$

Here $\Delta_{x}$ is the Laplace operator in the coordinates of $x, \partial_{\nu}$ is the outward normal derivative defined almost everywhere on $\partial \Omega$ e while $\partial_{\nu}=\partial_{z}=\partial / \partial z$ on $\Gamma$. Moreover, $\varphi$ is the velocity potential, $\lambda=\omega^{2} / g$ is a spectral parameter with the oscillation frequency $\omega>0$ and the acceleration $g$ due to gravity. The right hand side $f$ will be an element of a suitable function space.

The weak formulation [16], of the problem (1.6)-(1.8) reads as

$$
\left(\nabla_{x} \varphi, \nabla_{x} \psi\right)_{\Omega}-\lambda(\varphi, \psi)_{\Gamma}=(f, \psi)_{\Omega}, \psi \in H^{1}(\Omega)
$$

where $\nabla_{x}=\operatorname{grad}$ in $x,(\cdot, \cdot) \Xi$ is the natural scalar product in the Lebesgue space $L^{2}(\Xi)$ and $H^{1}(\Omega)$ is the Sobolev space. In case $f=0(1.9)$ can be interpreted as a standard spectral problem of a bounded, symmetric, therefore self-adjoint, linear operator in a Hilbert space $\mathcal{H}$, the so called Maz'ya space. We include in the appendix, Section A, the description of the spectrum of this operator, see Theorem A.1. 
However, to pose the radiation conditions related to the problem (1.6-1.8) we need to use appropriate weighted spaces $V_{\beta}^{1}(\Omega), \beta \in \mathbb{R}$, of Kondratiev type (see [11,12,35]) and to study the problem operator in these spaces. These spaces will be used throughout the paper to describe the asymptotical behaviour of functions, by presenting them as sums of explicit terms and remainders belonging to some $V_{\beta}^{1}(\Omega)$ with a small $\beta$. By $V_{\beta}^{1}(\Omega)$ we mean the completion of $C_{c}^{\infty}(\bar{\Omega} \backslash \mathcal{O})$ (infinitely smooth functions with supports separated from $\mathcal{O}$ ) with respect to the norm

$$
\left\|u ; V_{\beta}^{1}(\Omega)\right\|=\left(\left\|\varrho^{\beta} \nabla_{x} u ; L^{2}(\Omega)\right\|^{2}+\left\|\varrho^{\beta-1} u ; L^{2}(\Omega)\right\|^{2}\right)^{1 / 2},
$$

where $\varrho(x)=|x|$ is the distance to the irregularity point $\mathcal{O}$. Notice that diminishing $\beta$ shrinks the space $V_{\beta}^{1}(\Omega)$. Higher order spaces $V_{\beta}^{k}(\Omega), k \geq 2$, are defined in the same way using the norm

$$
\left\|u ; V_{\beta}^{k}(\Omega)\right\|^{2}=\sum_{l=0}^{k}\left\|\varrho^{\beta-k+l} \nabla_{x}^{l} u ; L^{2}(\Omega)\right\|^{2} .
$$

If $\Omega^{\prime}$ is a subdomain of $\Omega$, the space $V_{\beta}^{1}\left(\Omega^{\prime}\right)$ is defined just by replacing $\Omega$ by $\Omega^{\prime}$; in particular the weight functions $\varrho^{\beta}$ and $\varrho^{\beta-1}$ in (1.10) remain the same.

Moreover, given $\sigma \in \mathbb{R}$ we mean by $V_{\sigma}^{1}\left(\mathbb{R}^{2}\right)$ the completion of $C_{c}^{\infty}\left(\mathbb{R}_{\bullet}^{2}\right)$ with respect to the weighted Kondratiev norm

$$
\left\|v ; V_{\sigma}^{1}\left(\mathbb{R}^{2}\right)\right\|=\left(\left\|\nabla_{y} v ; L_{\sigma}^{2}\left(\mathbb{R}^{2}\right)\right\|^{2}+\left\|v ; L_{\sigma-1}^{2}\left(\mathbb{R}^{2}\right)\right\|^{2}\right)^{1 / 2}
$$

where the norm of the weighted Lebesgue space $L_{\sigma}^{2}\left(\mathbb{R}^{2}\right) \ni u$ is $\left\|r^{\sigma} u ; L^{2}\left(\mathbb{R}^{2}\right)\right\|$; recall $r=|y|$. If $D$ is a subdomain of $\mathbb{R}^{2}$, the space $V_{\sigma}^{1}(D)$ is again defined by replacing $\mathbb{R}^{2}$ by $D$ here.

The following trace inequality holds in $V_{\beta}^{1}(\Omega)$ :

Lemma 1.1. For any function $u \in C_{c}^{\infty}(\bar{\Omega} \backslash \mathcal{O})$, the inequality

$$
\left\|u ; L_{\beta}^{2}(\Gamma)\right\|:=\left\|\varrho^{\beta} u ; L^{2}(\Gamma)\right\| \leq c\left\|u ; V_{\beta}^{1}(\Omega)\right\|
$$

is valid, and the constant $c$ depends on $\Omega, \Gamma$ and $\beta$ only.

Proof. The inequality (1.13) with $\beta=0$ is proven in [38], Lemma 3.1 (see also [22], Sect. 5.4). If $\beta \neq 0$, we set $u_{\beta}=\varrho^{\beta} u$ and observe that

$$
\left\|\nabla_{x} u_{\beta} ; L^{2}(\Omega)\right\|^{2} \leq 2\left\|\varrho^{\beta} \nabla_{x} u ; L^{2}(\Omega)\right\|^{2}+c_{\beta}\left\|\varrho^{\beta-1} u ; L^{2}(\Omega)\right\|^{2} \leq c\left\|u ; V_{\beta}^{1}(\Omega)\right\|^{2}
$$

in order to conclude (1.13).

A solution of the problem (1.6-1.8) in the space $V_{\beta}^{1}(\Omega)$ means a function $\varphi$ satisfying

$$
\left(\nabla_{x} \varphi, \nabla_{x} \psi\right)_{\Omega}-\lambda(\varphi, \psi)_{\Gamma}=F(\psi)
$$

for all $\psi \in V_{-\beta}^{1}(\Omega)$, where $F \in V_{-\beta}^{1}(\Omega)^{*}$ is the linear functional $\psi \mapsto(f, \psi)_{\Omega}$ on the space $V_{-\beta}^{1}(\Omega)$. Owing to definition (1.10) and the trace inequality (1.13), the left hand side of (1.14) is properly defined for any $\varphi \in V_{\beta}^{1}(\Omega)$ and gives rise to a functional on $V_{-\beta}^{1}(\Omega) \ni \psi$. Thus, the left hand side of (1.14) determines the continuous mapping

$$
A_{\beta}(\lambda): V_{\beta}^{1}(\Omega) \rightarrow V_{-\beta}^{1}(\Omega)^{*}
$$


The main objective in Chapter 3 becomes to establish a criterion for the Fredholm property of $A_{\beta}(\lambda)$ and to calculate its index

$$
\operatorname{Ind} A_{\beta}(\lambda)=\operatorname{dim} \operatorname{ker} A_{\beta}(\lambda)-\operatorname{dim} \operatorname{coker} A_{\beta}(\lambda)
$$

where $\operatorname{ker} A_{\beta}(\lambda)$ denotes the kernel of the operator $A_{\beta}(\lambda)$ and

$$
\operatorname{coker} A_{\beta}(\lambda)=\operatorname{ker} A_{-\beta}(\lambda)
$$

because $A_{-\beta}(\lambda)$ is obviously the adjoint of $A_{\beta}(\lambda)$.

\subsection{Preliminary description of the results and the approach}

Chapter 2 contains an analysis of the asymptotic behaviour around $\mathcal{O}$ of functions in $V_{\beta}^{1}(\Omega)$, which are solutions of the problem (1.14). Given $\beta$ and a function $\varphi \in V_{\beta}^{1}(\Omega)$ we decompose it in a neighbourhood of $\mathcal{O}$ as

$$
\varphi(y, z)=\bar{\varphi}(y)+\varphi_{\perp}(y, z)
$$

where $\bar{\varphi}$ is the mean value function

$$
\bar{\varphi}(y)=h(y)^{-1} \int_{-h(y)}^{0} \varphi(y, z) \mathrm{d} z, \text { and } \int_{-h(y)}^{0} \varphi_{\perp}(y, z) \mathrm{d} z=0 .
$$

We analyze the decay properties of these components in terms of weighted norm estimates; as for $\varphi_{\perp}$, it will be shown by a few steps that it belongs to $V_{\beta_{1}}^{1}(\Omega)$, where $\beta_{1} \geq \beta-1 / 2$. As for $\bar{\varphi}$, its properties are studied with help of a limit partial differential equation in two variables $\left(y_{1}, y_{2}\right)$. This is derived in Sections 2.1-2.4 as a formal limit equation (2.7), using formal asymptotics in the vicinity of $\mathcal{O}$ and standard ansätze in the theory of thin domains ( $c f$. [26]). The properties of the solutions of (2.7) are found by an application of the Kondratiev theorem (see [11] and Thm. 2.2). These are transformed into the the main result of Chapter 2 (Thm. 2.11) on the asymptotic behaviour of $\varphi$ as $x \rightarrow \mathcal{O}$ : given $\beta$, a solution $\varphi \in V_{\beta}^{1}\left(\Xi_{R}\right)$ of the problem (1.6)-(1.8) can be written as the sum of $\tilde{\varphi} \in V_{\beta_{1}}^{1}\left(\Xi_{R}\right)$, where $\beta_{1} \in(\beta-1 / 2, \beta)$, plus a finite number of rather explicit functions called $w^{(j)} \in V_{\beta}^{1}\left(\Xi_{R}\right)$.

The number of functions $w^{(j)}$ depend on the parameter $\lambda$; we shall find increasing sequences $\left(\lambda_{q}^{\dagger}\right)_{q=1}^{\infty}$ and $\left(N_{q}\right)_{q=1}^{\infty}$ such that there are $2 N_{q}$ functions $w^{(j)}$ for $\lambda \in\left[\lambda_{q-1}^{\dagger}, \lambda_{q}^{\dagger}\right)$. In fact the functions $w^{(j)}$ emerge from the power-law solutions of the limit equation (2.7),

$$
v(y)=r^{\Lambda} V(\vartheta)
$$

where $\Lambda \in \mathbb{C}$ is a complex exponent, $V$ is a smooth function on the unit circle $\mathbb{S}^{1}$, and $(r, \vartheta)$ are the polar coordinates for $y$.

In Chapter 3 we examine the operator (1.15) of the problem (1.14) and establish in Theorem 3.2 a necessary and sufficient condition for the Fredholm property of $A_{\beta}(\lambda)$. It will be necessary to verify the estimate

$$
\left\|\varphi ; V_{\beta}^{1}(\Omega)\right\| \leq c_{\beta}\left(\left\|F ; V_{-\beta}^{1}(\Omega)^{*}\right\|+\left\|\varphi ; L_{\beta-1 / 2}^{2}(\Omega)\right\|\right)
$$

under a condition for $\beta$ and $\lambda$; in view of the compactness of the embedding $V_{\beta}^{1}(\Omega) \subset L_{\beta-1 / 2}^{2}(\Omega)$, this ensures the closedness of the range $\operatorname{Im} A_{\beta}(\lambda)$, the finite dimensionality of the subspace $\operatorname{ker} A_{\beta}(\lambda)$ and, moreover, the fact that

$$
\operatorname{dim} \operatorname{coker} A_{\beta}(\lambda)=\operatorname{dim} \operatorname{ker} A_{-\beta}(\lambda)<\infty
$$


In the last part of Chapter 3 we compute the index (1.16). The index formula (3.34) will involve the number $N_{q}$ of the above mentioned power-law solutions and thus depends on the spectral parameter $\lambda$. Let us describe the difference between the properties of the operator $A_{\beta}(\lambda)$ for $\lambda$ below and above the first threshold $\lambda_{\dagger}:=\lambda_{1}^{\dagger}$ (cf. (2.23)). In the case $\lambda \in\left[0, \lambda_{\dagger}\right)$ the operator $A_{0}(\lambda)$ is Fredholm and self-adjoint in the Kondratiev space $V_{0}^{1}(\Omega)$, which for $\beta=0$ coincides with the Sobolev space $H^{1}(\Omega)$. In other words, a solution $\varphi \in H^{1}(\Omega)$ with finite energy

$$
\int_{\Omega}\left|\nabla_{x} \varphi(x)\right|^{2} \mathrm{~d} x+\int_{\Gamma}|\varphi(x)|^{2} \mathrm{~d} y
$$

exists, provided the right-hand side $F \in H^{1}(\Omega)^{*}$ of $(1.9)$, or (1.14), satisfies the compatibility conditions

$$
F\left(\varphi_{j}\right)=0, j=1, \ldots, J(\lambda)
$$

where $\varphi_{1}, \ldots, \varphi_{J(\lambda)} \in H^{1}(\Omega)$ are solutions of the homogeneous $(F=0)$ problem (1.14). This solution $\varphi$ is defined up to a linear combination of $\varphi_{1}, \ldots, \varphi_{J(\lambda)}$. Under the orthogonality conditions

$$
\int_{\Omega} \varphi(x) \varphi_{j}(x) \mathrm{d} x=0, j=1, \ldots, J(\lambda),
$$

it becomes unique and admits the estimate

$$
\left\|\varphi ; H^{1}(\Omega)\right\| \leq c(\lambda)\left\|F ; H^{1}(\Omega)^{*}\right\|
$$

All the above facts are due to the Fredholm property of $A_{0}(\lambda)$. Notice that for $\lambda \in\left[0, \lambda_{\dagger}\right)$, also the operators $A_{\beta}(\lambda)$ are of Fredholm index 0 , if $|\beta|$ is small enough. Moreover, for $\beta$ large enough the index becomes positive while for sufficiently large $-\beta$ it is negative.

In the case $\lambda \geq \lambda_{\dagger}$ the operator $A_{0}(\lambda)$ is no longer Fredholm (and no other operator $A_{\beta}(\lambda)$ has index 0 , although almost all of them are Fredholm). We shall show that the range $\operatorname{Im} A_{0}(\lambda)=A_{0}(\lambda) H^{1}(\Omega)$ is not closed, though the kernel and cokernel are finite dimensional. This means that the orthogonality conditions (1.24) imply the uniqueness of the solution, but the estimate (1.25) does not hold.

The reason for losing the Fredholm property is but the appearance of the power-law solutions (1.20) with the exponents

$$
\Lambda=-1 \pm \mathrm{i} \varkappa_{n}(\lambda), n=1, \ldots, N
$$

where the numbers $\varkappa_{n}(\lambda)$ are nonnegative. The distinguishing feature of these solutions is that the integrals (1.22) diverge logarithmically at $\mathcal{O}$ (see Rem. 2.1).

Finally, in Chapter 4 we derive the radiation conditions for the water-wave problem from the requirement that the operator $A_{\beta}(\lambda)$ becomes Fredholm by choosing its domain and range carefully. First, the right-hand side of $F$ is to be taken from a smaller space $V_{\beta}^{1}(\Omega)^{*} \subset H^{1}(\Omega)^{*}$ with $\beta>0$. Second, a solution to (1.14), $\varphi$, is to be found in a bigger space $V_{\beta}^{1}(\Omega) \supset H^{1}(\Omega)$. Third, examining this solution we notice that it is defined up to a linear combination of the $J(\lambda)+N(\lambda)$ solutions $\varphi_{1}, \ldots, \varphi_{J(\lambda)} \in \operatorname{ker} A_{-\beta}(\lambda) \subset H^{1}(\Omega)$ and $\varphi_{J(\lambda)+1}, \ldots, \varphi_{J(\lambda)+N(\lambda)} \in V_{\beta}^{1}(\Omega)$ to the homogeneous problem (see (1.26); our result on Ind $A_{\beta}(\lambda)$ is crucial here). The final step is to formulate $N(\lambda)$ conditions which make the solution defined up to a linear combination of $\varphi_{1}, \ldots, \varphi_{J(\lambda)}$ only. The latter form a basis in the subspace coker $A_{\beta}(\lambda)$ and thus the operator involving these conditions becomes of index zero. In other words, we fix appropriately the $N(\lambda)$ coefficients in the abovementioned linear combinations, and these conditions are usually called radiation conditions. 
Our method involves a symplectic form, which naturally divides the waves in the water blanket $\Xi_{R}$ into incoming and outgoing ones. In the above explained procedure we actually allow only the outgoing waves: $A_{\beta}(\lambda)$ is made Fredholm and of index zero by restricting its domain to a subspace of $V_{\beta}^{1}(\Omega)$ which is a sum of functions in $V_{-\beta}^{1}(\Omega)$ and linear combinations of outgoing waves (see Thm. 4.2).

In the case all $\varkappa_{n}(\lambda)$ in (1.26) are positive, the imposed radiation conditions are physical (see explanations in Sect. 4.5). However, for $\lambda=\lambda_{\dagger}$ and for some $\lambda>\lambda_{\dagger}$ there appear power-logarithmic solutions (1.20) and our radiation conditions become intrinsic.

The appendix contains Theorem A.1 concerning the spectrum of the operator of the water-wave problem. It is a consequence of Theorem 3.2 .

We close this section with a review of related literature. First we mention a related paper [3], where trapped water-waves are considered in a cuspidal domain in dimension two. However, this corresponds to the situation where the cusp is less sharp, i.e., $H(y)=|y|^{\alpha}$ with $\alpha<2$ and thus the compactness of the trace mapping still holds. Concerning Chapter 2, although the theory of elliptic problems in domains with piecewise smooth boundaries is well understood (see e.g. $[11-13,20,21,27,35]$ ), there is no general method to investigate boundary value problems in "sharp" cuspidal domains, except in the case of peak-shaped domain, Figure 1 (see $[12,19,40]$ ). In the present paper, the irregularity point with tangency of two boundary components (Fig. 3) results in the thinning water blanket $\Omega \cap \mathcal{U}$, which is cuspidal in the $z$-direction but conical in the two other directions $y_{1}$ and $y_{2}$. This complication of geometry changes crucially the asymptotic behaviour of solutions as $x \rightarrow \mathcal{O}$, and results have been obtained only for particular problems in mathematical physics, namely, for the Neumann problem for a scalar second-order elliptic equation (see [24]), the Stokes equations (see [25]) and the elasticity system with traction-free boundary conditions (see [36]). Therefore, we are forced to start our study in Chapter 2 by deriving the asymptotic representations for a solution $\varphi \in V_{\beta}^{1}(\Omega)$ of the problem (1.6-1.8).

As for the radiation conditions, there are several known methods for composing them, but this paper is certainly the first one where the wave phenomenon in a finite volume is treated rigorously. The situation found in Chapter 3 is typical in diffraction problems related to wave phenomena, and our way to supply the problem with a Fredholm operator is in some sense standard. The symplectic form, which we use here and which divides the waves around $\mathcal{O}$ into incoming and outgoing ones, is inspired by the approach proposed in [34] (see also [35], Chap. 6). This is among the the most general and flexible. The calculation of the Poynting vector is based on the derived asymptotic forms, and it is crucial for the explanation of the physical nature of the phenomena.

\section{Asymptotic ANALYsis}

In the main result, Theorem 2.11, of this section we show that assuming $\beta$ and a solution $\varphi \in V_{\beta}^{1}(\Omega)$ given, $\varphi$ can be written as a sum of finitely many quite explicit functions $w^{(q)} \in V_{\beta}^{1}(\Omega)$ and a term $\tilde{\varphi}$, which has a better decay at $\mathcal{O}$, as a function in $V_{\beta_{1}}^{1}(\Omega)$, where $\beta-1 / 2 \leq \beta_{1}<\beta$.

Chapter 2 is divided into two entities. Sections 2.1-2.4 contain a formal derivation of a limit equation and its analysis, by which the functions $w^{(q)}$ are found in Theorem 2.2. Sections 2.5-2.7 include the analysis of the asymptotic behaviour of elements of $V_{\beta}^{1}(\Omega)$ around $\mathcal{O}$ and the main result of Chapter 2, Theorem 2.11.

\subsection{The formal asymptotics}

We seek for a formal solution of (1.6)-(1.8), $f=0$, which takes in the vicinity of the point $\mathcal{O}$ the form

$$
\varphi(x)=v(y)+h(y) s\left(y, h(y)^{-1} z\right)+\ldots
$$

Here, the dots stand for lower-order terms, and moreover, in the polar coordinates $(r, \vartheta)$ of the $y$-plane, $v(y)=$ $r^{\Lambda} V(\vartheta)$ is a power law solution (1.20) of the limit differential equation (2.7) (to be derived), and

$$
s(y, \zeta)=r^{\Lambda} S(\vartheta, \zeta), S \in C^{2}\left(\mathbb{S}^{1} \times[-1,0]\right) .
$$


By $\zeta=h(y)^{-1} z$ we understand the fast variable in the water blanket $\Xi_{R}$, see (2.22). The possible values of $\Lambda \in \mathbb{C}$ will be later determined from the eigenvalues of the limit equation (2.7), see (2.12) and (2.10).

In the coordinates $y, \zeta$ the differential operators $\Delta_{x}$ and $\partial_{\nu}$ are given by

$$
\begin{aligned}
& \Delta_{x}=h(y)^{-2} \partial_{\zeta}^{2}+\left(\nabla_{y}-h(y)^{-1} \nabla_{y} h(y) \zeta \partial_{\zeta}\right)^{2}, \\
& \partial_{\nu}=-\left(1+\left|\nabla_{y} h(y)\right|^{2}\right)^{-1 / 2}\left(h(y)^{-1} \partial_{\zeta}+\nabla_{y} h(y) \cdot \nabla_{y}\right) .
\end{aligned}
$$

Here $\partial_{\zeta}=\partial / \partial \zeta, \nabla_{y}=$ grad in the variables $y=\left(y_{1}, y_{2}\right)$ and the central dot stands for the scalar product in $\mathbb{R}^{2}$. Notice that the formula (2.4) follows from the definition (1.5).

We insert the decompositions (2.1) and (2.3), (2.4) into the equation (1.6) and into the boundary conditions (1.7), (1.8) in the vicinity of the point $\mathcal{O}$. Then, $h(y)$ is replaced by $H(y)$, see (1.3). Finally we collect the terms of order $r^{\operatorname{Re} \Lambda-2}$ in (1.6) and $r^{\operatorname{Re} \Lambda}$ in (1.7), (1.8), and arrive at the relations

$$
\begin{aligned}
-H(y)^{-1} \partial_{\zeta}^{2} s(y, \zeta) & =\Delta_{y} v(y), \zeta \in(-1,0) \\
-\partial_{\zeta} s(y,-1) & =\nabla_{y} H(y) \cdot \nabla_{y} v(y), \quad \partial_{\zeta} s(y, 0)=\lambda v(y) .
\end{aligned}
$$

The compatibility condition in the Neumann problem (2.5) for the ordinary differential equation in the interval $(-1,0)$ reads as

$$
\begin{aligned}
-H(y) \Delta_{y} v(y) & =\int_{-1}^{0} \partial_{\zeta}^{2} s(y, \zeta) \mathrm{d} \zeta=\left[\partial_{\zeta} s(y, \zeta)\right]_{-1}^{0} \\
& =\lambda v(y)+\nabla_{y} H(y) \cdot \nabla_{y} v(y) .
\end{aligned}
$$

In other words, to find the second term in the asymptotic expansion (2.1) we need to solve the partial differential equation

$$
-\nabla_{y} \cdot H(y) \nabla_{y} v(y)-\lambda v(y)=0, \quad y \in \mathbb{R}_{\bullet}^{2}
$$

Note that, owing to (1.4), the principal part of the differential operator $-\nabla_{y} \cdot H(y) \nabla_{y}$ degenerates at $y=0$.

\subsection{Power-law solutions of the limit equation}

The operator $\nabla_{y} \cdot H(y) \nabla_{y}$ reads in the polar coordinates as

$$
-r^{-1} \partial_{r} r^{3} \mathcal{H}(\vartheta) \partial_{r}-r^{-2} \partial_{\vartheta} r^{2} \mathcal{H}(\vartheta) \partial_{\vartheta}
$$

where

$$
H(y)=r^{2} \mathcal{H}(\vartheta), \mathcal{H} \in C^{\infty}\left(\mathbb{S}^{1}\right), \mathcal{H}>0 .
$$

We proceed by usual separation of variables and insert this into (2.7); denoting

$$
\mu=(\Lambda+2) \Lambda+1=(\Lambda+1)^{2}
$$

we obtain the ordinary differential equation

$$
-\frac{\partial}{\partial \vartheta} \mathcal{H}(\vartheta) \frac{\partial V}{\partial \vartheta}(\vartheta)+\mathcal{H}(\vartheta) V(\vartheta)-\lambda V(\vartheta)=\mu \mathcal{H}(\vartheta) V(\vartheta), \vartheta \in \mathbb{S}^{1} .
$$


The variational formulation of the spectral problem (2.11) involves the closed semibounded below quadratic form

$$
q(U, V ; \lambda)=\left(\mathcal{H} \partial_{\vartheta} V, \partial_{\vartheta} U\right)_{\mathbb{S}^{1}}+(\mathcal{H} V, U)_{\mathbb{S}^{1}}-\lambda(V, U)_{\mathbb{S}^{1}},
$$

where $(\cdot, \cdot)_{\mathbb{S}^{1}}$ denotes the inner product of $L^{2}\left(\mathbb{S}^{1}\right)$. By [2], Theorem 10.2.2, the spectral problem (2.11) admits for any $\lambda \in \mathbb{R}^{+}$the monotone unbounded sequence of eigenvalues

$$
\mu_{1}(\lambda) \leq \mu_{2}(\lambda) \leq \ldots \leq \mu_{p}(\lambda) \leq \ldots,
$$

which are listed taking into account their multiplicites. Moreover, there exists a strictly monotone unbounded sequence of thresholds

$$
0=: \lambda_{0}^{\dagger}<\lambda_{\dagger}:=\lambda_{1}^{\dagger}<\lambda_{2}^{\dagger}<\ldots<\lambda_{q}^{\dagger}<\ldots
$$

such that, in the case $\lambda \in\left[\lambda_{q-1}^{\dagger}, \lambda_{q}^{\dagger}\right)$, the number of non-positive eigenvalues in (2.12) is $N_{q}$ and

$$
0=N_{1}<N_{2}<\ldots<N_{q}<\ldots
$$

In other words, for $\lambda \in\left[\lambda_{q-1}^{\dagger}, \lambda_{q}^{\dagger}\right)$,

$$
\mu_{1}(\lambda) \leq \ldots \leq \mu_{N_{q}}(\lambda) \leq 0<\mu_{N_{q}+1}(\lambda) \leq \ldots
$$

Note that the multiplicity of an eigenvalue of an elliptic ordinary second-order differential equation on the circle $\mathbb{S}^{1}$ cannot exceed 2. Hence, $N_{q+1} \leq N_{q}+2$ in (2.14).

It is instructive to consider the special case of axially symmetric cusp, since then one can calculate the above numbers; this is done in the next section for the convenience of the reader.

Eigenfunctions corresponding to the eigenvalues (2.12) are denoted by $V_{1}, V_{2}, \ldots, V_{p}, \ldots$ They can be subject to the orthogonality and normalization conditions

$$
\left(\mathcal{H} V_{p}, V_{q}\right)_{\mathbb{S}^{1}}=\delta_{p, q}, p, q \in \mathbb{N},
$$

where $\delta_{p, q}$ is Kronecker's symbol.

Owing to (2.10), a positive eigenvalue $\mu_{j}(\lambda)>0$ gives rise to the following couple of power-law solutions

$$
v_{j}^{ \pm}(y)=r^{\Lambda_{j}^{ \pm}} V_{j}(\vartheta), \Lambda_{j}^{ \pm}=-1 \pm \mu_{j}(\lambda)^{1 / 2},
$$

with the real exponents $\Lambda_{j}^{ \pm}=\Lambda_{j}^{ \pm}(\lambda)$ depending on the parameter $\lambda$. If $\mu_{j}(\lambda)<0$, the exponents $\Lambda_{j}^{ \pm}=\Lambda_{j}^{ \pm}(\lambda)$ in the power-law solutions

$$
v_{j}^{ \pm}(y)=r^{\Lambda_{j}^{ \pm}} V_{j}(\vartheta), \Lambda_{j}^{ \pm}=-1 \pm \mathrm{i}\left|\mu_{j}(\lambda)\right|^{1 / 2}
$$

become complex. In the case $\mu_{j}(\lambda)=0$, only one power-law solution

$$
v_{j}^{0}(y)=r^{-1} V_{j}(\vartheta)
$$

appears; however, a direct calculation demonstrates that the function

$$
v_{j}^{1}(y)=r^{-1} \ln r V_{j}(\vartheta),
$$

which is linear in $\ln r$, satisfies the differential equation (2.7) as well. 
The functions in (2.17)-(2.20) are denoted in the sequel by $w^{(j)}$; see e.g. Theorem 2.2 .

Ignoring the factor $r^{-1}$ we shall call the solutions (2.18) oscillating and the solutions (2.17) decaying (plus) or growing (minus). The solutions (2.19) and (2.20) remain unnamed since we only employ their linear combinations in the sequel.

Remark 2.1. The functions (2.18-2.20), defined in the water blanket (1.5), do not belong to the Sobolev space, because the integral

$$
\int_{\mathbb{B}_{R}} \int_{h(y)}^{0}\left|\partial_{r} r^{-1 \pm \mathrm{i}\left|\mu_{j}(\lambda)\right|} V(\vartheta)\right|^{2} \mathrm{~d} z \mathrm{~d} y \cong \int_{\mathbb{S}^{1}} \mathcal{H}(\vartheta)|V(\vartheta)|^{2} \mathrm{~d} \vartheta \int_{0}^{R} r^{2}\left(r^{-2}\right)^{2} r \mathrm{~d} r
$$

diverges.

\subsection{Axially symmetric case}

An orthogonal transform of the coordinates $y$ turns the quadratic polynomial (1.4) into

$$
H(y)=H_{1} y_{1}^{2}+H_{2} y_{2}^{2}, H_{p}>0 .
$$

If the submerged body is axially symmetric, the identity

$$
H_{1}=H_{2}=: H_{0}>0
$$

holds, and the solutions of the spectral problem (2.11) are the functions

$$
V(\vartheta)=\mathrm{e}^{ \pm \mathrm{i} k \vartheta}
$$

corresponding to eigenvalues

$$
\mu_{2 k}=\mu_{2 k+1}=M_{ \pm k}:=k^{2}+1-\frac{\lambda}{H_{0}}, k=0,1,2, \ldots
$$

The eigenvalue $\mu_{1}=M_{0}$ is thus simple and the other eigenvalues $\mu_{2 j}=\mu_{2 j+1}, j \in \mathbb{N}=\{1,2,3, \ldots\}$, are of multiplicity 2. If $\lambda \in\left[H_{0}\left((q-1)^{2}+1\right), H_{0}\left(q^{2}+1\right)\right)$ and $q \in \mathbb{N}$, there exist $2 q-1$ non-positive eigenvalues $M_{0}, \ldots, M_{ \pm(q-1)}$ in (2.17). Hence, in this case, $\lambda_{1}^{\dagger}=H_{0}=\lambda_{\dagger}$, and, for all $q \geq 2$,

$$
\lambda_{q}^{\dagger}=H_{0}\left((q-1)^{2}+1\right), \quad N_{q}=2 q-3 .
$$

\subsection{Some facts about the limit equation}

We regard the punctured plane $\mathbb{R}_{\bullet}^{2}$ as the full angle and apply results of the theory of elliptic problems in domains with conical boundary points (see the key works [11,20,21,27] and, e.g., monographs [12,35]) in order to gain information on the solvability of the limit equation (2.7) and asymptotics of its solution. We apply this theory to the operator

$$
\mathcal{L}_{\beta}: V_{1+\beta}^{1}\left(\mathbb{R}^{2}\right) \rightarrow V_{1-\beta}^{1}\left(\mathbb{R}^{2}\right)^{*},
$$

which is determined (analogously to (1.14)-(1.15)) by the weak formulation

$$
\left(H \nabla_{y} v, \nabla_{y} u\right)_{\mathbb{R}^{2}}-\lambda(v, u)_{\mathbb{R}^{2}}=G(u), u \in V_{1-\beta}^{1}\left(\mathbb{R}^{2}\right) .
$$


of the differential equation

$$
-\nabla_{y} \cdot H(y) \nabla_{y} v(y)-\lambda v(y)=g(y), y \in \mathbb{R}_{\bullet}^{2}
$$

Here $V_{\sigma}^{1}\left(\mathbb{R}^{2}\right)$ is defined in (1.12). Note that $V_{1+\beta}^{1}\left(\mathbb{R}^{2}\right) \subset L_{\beta}^{2}\left(\mathbb{R}^{2}\right)=L_{-\beta}^{2}\left(\mathbb{R}^{2}\right)^{*}$ and $V_{1-\beta}^{1}\left(\mathbb{R}^{2}\right) \subset L_{-\beta}^{2}\left(\mathbb{R}^{2}\right)$ so that the terms on the left-hand side of (2.25) involve extensions of the natural scalar product of $L^{2}\left(\mathbb{R}^{2}\right)$ to the duality between appropriate weighted spaces $L_{\sigma}^{2}\left(\mathbb{R}^{2}\right)$ and $L_{-\sigma}^{2}\left(\mathbb{R}^{2}\right)$. In the same way, for $g \in L_{\beta}^{2}\left(\mathbb{R}^{2}\right)$, $G(u)=(g, u)_{\mathbb{R}^{2}}$ is a continuous functional in $V_{1-\beta}^{1}\left(\mathbb{R}^{2}\right) \ni u$. In the sequel we of course consider arbitrary functionals $G \in V_{1-\beta}^{1}\left(\mathbb{R}^{2}\right)^{*}$.

Theorem 2.2 ([11]; see also [35], Thm. 3.1.1 and 3.1.4).

(1) The operator $\mathcal{L}_{\beta}$ in (2.24) is an isomorphism, if and only if

$$
\beta+1+\operatorname{Re} \Lambda_{j}^{ \pm} \neq 0 \text { for any } j \in \mathbb{N},
$$

where the exponents $\Lambda_{j}^{ \pm}$are determined in (2.17-2.20). If (2.27) is violated, the operator $\mathcal{L}_{\beta}$ is not Fredholm, since the range $\operatorname{Im} \mathcal{L}_{\beta}$ is not a closed subspace of $V_{-\beta}^{1}\left(\mathbb{R}^{2}\right)^{*}$.

(2) Let the condition (2.27) be fulfilled for $\beta_{1}$ and $\beta_{2}$ with $\beta_{2}>\beta_{1}$. If $G \in V_{1-\beta_{1}}^{1}\left(\mathbb{R}^{2}\right)^{*} \cap V_{1-\beta_{2}}^{1}\left(\mathbb{R}^{2}\right)^{*}$, the solutions $v_{p} \in V_{1+\beta_{p}}^{1}\left(\mathbb{R}^{2}\right)$ with $p=1,2$, given by the first assertion, satisfy

$$
v_{2}(y)=v_{1}(y)+\sum_{j} c^{(j)} w^{(j)}(y)
$$

where the summation is performed over all power-law solutions (2.17-2.20) with the exponents $\Lambda_{j}$ satisfying the inclusion

$$
\operatorname{Re} \Lambda_{j} \in\left(-1-\beta_{2},-1-\beta_{1}\right)
$$

The coefficients $c^{(j)}$ in (2.28) meet the estimate

$$
\sum_{q}\left|c^{(j)}\right| \leq c\left(\left\|G ; V_{1-\beta_{1}}^{1}\left(\mathbb{R}^{2}\right)^{*}\right\|+\left\|G ; V_{1-\beta_{2}}^{1}\left(\mathbb{R}^{2}\right)^{*}\right\|\right),
$$

where the constant $c$ depends on $\beta_{2}$ and $\beta_{1}$ but not on $G$.

Remark 2.3. Since always $\Lambda_{j}^{+}+\Lambda_{j}^{-}=-2$, the weight indices $\pm \beta$ satisfy the condition (2.27) simultaneously.

A simple explanation of the method of [11] can be found for example in Chapter 2 of [35], see also the introductory chapters of the books $[12,13]$. We emphasize that formula (2.27) gives the asymptotics for the solution $\varphi_{2} \in V_{1+\beta_{2}}^{1}\left(\mathbb{R}^{2}\right)$ as $r \rightarrow+0$ and at the same time also for the solution $\varphi_{1} \in V_{1+\beta_{1}}^{1}\left(\mathbb{R}^{2}\right)$ as $r \rightarrow+\infty$. Only the first of these is interesting for the present study. The terms $c^{(j)} w^{(j)}$ in $(2.28)$ belong to $V_{1+\beta_{2}}^{1}\left(\mathbb{B}_{R}\right)$ for any finite $R$ but live outside $V_{1+\beta_{1}}^{1}\left(\mathbb{B}_{R}\right)$. The number of such terms equals $2 N_{q}$, according to the definitions in Section 2.2.

\subsection{Decomposing the solution}

In Sections 2.5-2.7 we consider the asymptotic behaviour of $\varphi \in V_{\beta}^{1}(\Omega)$ by treating the components $\bar{\varphi}$ and $\varphi_{\perp}$, see (1.19), separately using their typical properties. Here, "asymptotic behaviour" means belonging to a weighted space, say $V_{\sigma}^{1}(\Omega)$, with an as good, i.e. small, $\sigma$ as possible. In particular, if $\varphi$ is a solution of (1.14), the results of Sections 2.1-2.4 will be applied to the mean function $\bar{\varphi}$ (it depends on two variables only), to separate a more slowly decaying, but explicit part, and the remainder which decays more rapidly. 
The component $\varphi_{\perp}$ will have a faster decay as a consequence of the orthogonality condition in (1.19). These ultimately lead to the main result, that is, Theorem 2.11 .

Throughout Section 2.5 we assume that $\beta \in \mathbb{R}$ is given, $\varphi$ is an arbitrary element of $V_{\beta}^{1}(\Omega)$, and the components $\bar{\varphi}$ and $\varphi_{\perp}$ are defined as in (1.19). The aim is to prove Lemmas 2.4-2.6, concerning asymptotic behaviour of $\bar{\varphi}$ and $\varphi_{\perp}$.

If the support of $\varphi$ is separated from $\mathcal{O}$, the function falls into $V_{\sigma}^{1}(\Omega)$ with any weight index $\sigma$. Therefore, we may assume that $\varphi$ is null outside the closure $\bar{\Xi}_{R}$ of the blanket (1.5). Otherwise we multiply $\varphi$ with a suitable cut-off function. The functions $\bar{\varphi}$ and $\varphi_{\perp}$ are defined in $\mathbb{B}_{R}$ and $\Xi_{R}$, respectively.

Lemma 2.4. Let $\varphi \in V_{\beta}^{1}(\Omega)$ satisfy $\operatorname{supp} \varphi \subset \bar{\Xi}_{R}$. The mean value function $\bar{\varphi}$ falls into the space $V_{1+\beta}^{1}\left(\mathbb{B}_{R}\right)$ and meets the estimate

$$
\left\|\bar{\varphi} ; V_{1+\beta}^{1}\left(\mathbb{B}_{R}\right)\right\| \leq c\left\|\varphi ; V_{\beta}^{1}\left(\Xi_{R}\right)\right\| .
$$

Proof. In view of (1.2-1.4) we get using the Schwartz inequality

$$
\begin{aligned}
\int_{\mathbb{B}_{R}} r^{2 \beta}|\bar{\varphi}(y)|^{2} \mathrm{~d} y & =\int_{\mathbb{B}_{R}} r^{2 \beta} h(y)^{-2}\left|\int_{-h(y)}^{0} \varphi(y, z) \mathrm{d} z\right|^{2} \mathrm{~d} y \\
& \leq \int_{\Xi_{R}} r^{2 \beta} h(y)^{-1}|\varphi(x)|^{2} \mathrm{~d} x \leq c\left\|\varphi ; L_{\beta-1}^{2}\left(\Xi_{R}\right)\right\|^{2} .
\end{aligned}
$$

Furthermore, let us write

$$
\begin{aligned}
\nabla_{y} \bar{\varphi}(y)= & \frac{1}{h(y)} \int_{-h(y)}^{0} \nabla_{y} \varphi(y, z) \mathrm{d} z-\frac{\nabla_{y} h(y)}{h(y)^{2}} \int_{-h(y)}^{0} \varphi(y, z) \mathrm{d} z \\
& +h(y)^{-1} \nabla_{y} h(y) \varphi(y,-h(y))=: Z_{1}(y)+Z_{2}(y)+Z_{3}(y)
\end{aligned}
$$

Recalling that $\left|\nabla_{y} h(y)\right| \leq c r$ and repeating the calculation (2.32), we obtain

$$
\begin{aligned}
& \left\|Z_{1} ; L_{1+\beta}^{2}\left(\mathbb{B}_{R}\right)\right\|^{2} \leq c\left\|\nabla_{y} \varphi ; L_{\beta}^{2}\left(\Xi_{R}\right)\right\|^{2}, \\
& \left\|Z_{2} ; L_{1+\beta}^{2}\left(\mathbb{B}_{R}\right)\right\|^{2} \leq c\left\|\varphi ; L_{\beta-1}^{2}\left(\Xi_{R}\right)\right\|^{2} .
\end{aligned}
$$

Finally, the Newton-Leibnitz formula

$$
\varphi(y,-h(y))=\varphi(y, z)-\int_{-h(y)}^{z} \partial_{\zeta} \varphi(y, \zeta) \mathrm{d} \zeta
$$

leads to the relations

$$
\begin{aligned}
\varphi(y,-h(y)) & =\frac{1}{h(y)} \int_{-h(y)}^{0} \varphi(y, z) \mathrm{d} z-\frac{1}{h(y)} \int_{-h(y)}^{0} \int_{-h(y)}^{z} \partial_{\zeta} \varphi(y, \zeta) \mathrm{d} \zeta \mathrm{d} z \\
& =\bar{\varphi}(y)+\frac{1}{h(y)} \int_{-h(y)}^{0} \zeta \partial_{\zeta} \varphi(y, \zeta) \mathrm{d} \zeta
\end{aligned}
$$


and

$$
\begin{aligned}
\int_{\mathbb{B}_{R}} r^{2(1+\beta)}\left|Z_{3}(y)\right|^{2} \mathrm{~d} y & \leq c\left(\int_{\mathbb{B}_{R}} r^{2(1+\beta)} \frac{\left|\nabla_{y} h(y)\right|^{2}}{|h(y)|^{2}}|\bar{\varphi}(y)|^{2} \mathrm{~d} y+\left.\int_{\mathbb{B}_{R}} r^{2(1+\beta)} \frac{\left|\nabla_{y} h(y)\right|^{2}}{|h(y)|^{4}} \int_{-h(y)}^{0} \zeta \partial_{\zeta} \varphi(y, \zeta) \mathrm{d} \zeta\right|^{2} \mathrm{~d} y\right) \\
& \leq c\left(\int_{\mathbb{B}_{R}} r^{2 \beta}|\bar{\varphi}(y)|^{2} \mathrm{~d} y+\int_{\Xi_{R}} r^{2 \beta}\left|\partial_{\zeta} \varphi(y, \zeta)\right|^{2} \mathrm{~d} y \mathrm{~d} \zeta\right) \\
& \leq C\left\|\varphi ; V_{\beta}^{1}\left(\Xi_{R}\right)\right\|^{2} .
\end{aligned}
$$

The latter completes the proof.

Lemma 2.5. Let $\varphi \in V_{\beta}^{1}(\Omega)$ satisfy $\operatorname{supp} \varphi \subset \bar{\Xi}_{R}$. The functions $\varphi^{+}(y)=\varphi(y, 0)-\bar{\varphi}(y)=\varphi_{\perp}(y, 0)$ and $\varphi^{-}(y)=\varphi(y,-h(y))-\bar{\varphi}(y)=\varphi_{\perp}(y,-h(y))$ belong to $L_{\beta-1}^{2}\left(\mathbb{B}_{R}\right)$ and satisfy

$$
\left\|\varphi^{ \pm} ; L_{\beta-1}^{2}\left(\mathbb{B}_{R}\right)\right\| \leq c\left\|\varphi ; V_{\beta}^{1}\left(\Xi_{R}\right)\right\|
$$

Proof. Based on (2.33), we obtain

$$
\begin{aligned}
\int_{\mathbb{B}_{R}} r^{2(\beta-1)}\left|\varphi^{-}(y)\right|^{2} \mathrm{~d} y & =\int_{\mathbb{B}_{R}} r^{2(\beta-1)}|h(y)|^{-2}\left|\int_{h(y)}^{0} \zeta \partial_{\zeta} \varphi(y, \zeta) \mathrm{d} \zeta\right|^{2} \mathrm{~d} y \\
& \leq c \int_{\Xi_{R}} r^{2(\beta-1)} h(y)\left|\partial_{z} \varphi(y, s)\right|^{2} \mathrm{~d} x \leq c\left\|\partial_{z} \varphi ; L_{\beta}^{2}(\Xi)\right\|^{2}
\end{aligned}
$$

Estimation of $\varphi^{+}$requires a similar argument.

Since $\partial_{z} \varphi_{\perp}(y, z)=\partial_{z} \varphi(y, z)$, the next simple assertion is established by the Poincaré inequality on the interval $(-h(y), 0)$, which is small as $r \rightarrow 0^{+}$.

Lemma 2.6. If $\varphi \in V_{\beta}^{1}(\Omega)$ and $\operatorname{supp} \varphi \subset \bar{\Xi}_{R}$, the function $\varphi_{\perp}$ belongs to $L_{\beta-2}^{2}\left(\Xi_{R}\right)$ and satisfies

$$
\left\|\varphi_{\perp} ; L_{\beta-2}^{2}\left(\Xi_{R}\right)\right\| \leq c\left\|\varphi ; V_{\beta}^{1}\left(\Xi_{R}\right)\right\|
$$

Proof. In view of the orthogonality condition (1.18) we have

$$
\begin{aligned}
\int_{\mathbb{B}_{R}} r^{2(\beta-2)} \int_{-h(y)}^{0}\left|\varphi_{\perp}(y, z)\right|^{2} \mathrm{~d} z \mathrm{~d} y & \leq \int_{\Xi_{R}} r^{2(\beta-2)}|h(y)|^{2}\left|\partial_{z} \varphi(x)\right|^{2} \mathrm{~d} x \\
& \leq c\left\|\partial_{z} \varphi ; L_{\beta}^{2}\left(\Xi_{R}\right)\right\|^{2} .
\end{aligned}
$$

\subsection{Dimension reduction}

The aim of Section 2.6 is to formulate equations for the components $\bar{\varphi}$ and $\varphi_{\perp}$ of a solution $\varphi \in V_{\beta}^{1}(\Omega)$ of the problem (1.14). Moreover, we shall establish for the mean function $\bar{\varphi}$ a connection to the limit equation (2.7), 
so that in the next section, Theorem 2.2 (in the form of Lem. 2.10) can be applied to it. Recall that $\bar{\varphi}$ is a function of $y$ only, being constant in $z$-direction.

So, in this section we assume that $\varphi \in V_{\beta}^{1}(\omega)$ solves (1.14) and that $F$ and the indices $\beta$ and $\beta_{1}$ satisfy

$$
F \in V_{-\beta_{1}}^{1}(\Omega)^{*} \subset V_{-\beta}^{1}(\Omega)^{*}, \beta_{1}<\beta_{2}=: \beta \leq \beta_{1}+1 / 2 .
$$

Here, the last inequality is assumed due to technical reasons, but it is not really essential, since for example in Theorem 2.11 one could apply the statement of the theorem iteratively step by step to diminish the weight index $\beta$.

Let $\chi \in C^{\infty}(\bar{\Omega})$ be a cut-off function which is constant in the variable $z$, vanishes outside $\bar{\Xi}_{R}$ and is equal to one inside the blanket (1.5) for $r \leq R / 2$.

Lemma 2.7. If $\varphi$ and $F$ are as above, the function $\chi \varphi$ solves (1.14) with a functional $\widehat{F}$, see (2.38), on the right hand side, $\widehat{F}$ still satisfying the assumptions (2.36). Moreover,

$$
\left\|\widehat{F} ; V_{-\beta_{1}}^{1}(\Omega)^{*}\right\| \leq c\left(\left\|F ; V_{-\beta_{1}}^{1}(\Omega)^{*}\right\|+\left\|\varphi ; V_{\beta}^{1}(\Omega)\right\|\right) .
$$

Proof. Let $\psi \in V_{-\beta}^{1}(\Omega)$ be an arbitrary test function and put the test function $\widehat{\psi}=\chi \psi \in V_{-\beta}^{1}(\Omega)$ into (1.14). The left hand side becomes

$$
\begin{array}{r}
\left(\nabla_{x} \varphi, \chi \nabla_{x} \psi\right)_{\Omega}+\left(\nabla_{x} \varphi, \psi \nabla_{x} \chi\right)_{\Omega}-\lambda(\varphi, \chi \psi)_{\Gamma}=\left(\nabla_{x}(\chi \varphi), \nabla_{x} \psi\right)_{\Omega}-\left(\varphi \nabla_{x} \chi, \nabla_{x} \psi\right)_{\Omega} \\
+\left(\nabla_{x} \varphi, \psi \nabla_{x} \chi\right)_{\Omega}-\lambda(\chi \varphi, \psi)_{\Gamma}
\end{array}
$$

so that the function $\chi \varphi$ indeed satisfies (1.14), if the functional $\widehat{F}$ on the right hand side is defined by

$$
\widehat{F}(\psi):=F(\chi \psi)-\left(\nabla_{x} \varphi, \psi \nabla_{x} \chi\right)_{\Omega}+\left(\varphi \nabla_{x} \chi, \nabla_{x} \psi\right)_{\Omega} .
$$

Notice that still $\widehat{F} \in V_{-\beta_{1}}^{1}(\Omega)^{*}$, since the support of $\nabla_{x} \chi$ belongs to the set $\left\{x \in \Xi_{R}: R>r>R / 2\right\}$. The norm estimate (2.37) follows from the definition.

In the sequel we redefine $\varphi$ as $\chi \varphi$. Also, given a test function $\psi \in V_{-\beta}^{1}(\Omega)$ we write $\psi=\bar{\psi}+\psi_{\perp}$ as in the decomposition (1.18).

The two integral identities of the following lemma are obtained just by inserting to (1.14) the decomposition (1.18) of $\varphi$ and $\psi$, since the components $\bar{\psi}$ and $\psi_{\perp}$ can be chosen independently. In addition, in the first term of $(2.39)$ we have integrated over $(-h(y), 0) \ni z$.

Lemma 2.8. If $\varphi$ is as above, the following equalities hold for all test functions $\psi \in V_{-\beta}^{1}(\Omega)$. First,

$$
\left(h \nabla_{y} \bar{\varphi}, \nabla_{y} \bar{\psi}\right)_{\mathbb{B}_{R}}-\lambda(\bar{\varphi}, \bar{\psi})_{\mathbb{B}_{R}}=\bar{G}(\bar{\psi}),
$$

where

$$
\bar{G}(\bar{\psi}):=\widehat{F}(\bar{\psi})-\left(\nabla_{y} \varphi_{\perp}, \nabla_{y} \bar{\psi}\right)_{\Xi_{R}}+\lambda\left(\left.\varphi_{\perp}\right|_{z=0}, \bar{\psi}\right)_{\mathbb{B}_{R}} .
$$

Second,

$$
\left(\nabla_{x} \varphi_{\perp}, \nabla_{x} \psi_{\perp}\right) \Xi_{R}=G_{\perp}\left(\psi_{\perp}\right)
$$

where

$$
G_{\perp}\left(\psi_{\perp}\right):=\widehat{F}\left(\psi_{\perp}\right)-\left(\nabla_{y} \bar{\varphi}, \nabla_{y} \psi_{\perp}\right)_{\Xi_{R}}+\lambda\left(\left.\varphi\right|_{z=0},\left.\psi_{\perp}\right|_{z=0}\right)_{\mathbb{B}_{R}} .
$$


Remark 2.9. We notice that, due to (1.19),

$$
0=\nabla_{y} \int_{-h(y)}^{0} \varphi_{\perp}(y, z) \mathrm{d} z=\int_{-h(y)}^{0} \nabla_{y} \varphi_{\perp}(y, z) \mathrm{d} z+\varphi_{\perp}(y,-h(y)) \nabla_{y} h(y)
$$

and, hence, by definition of $\varphi^{-}$in Lemma 2.5, we have

$$
\begin{aligned}
\left(\nabla_{y} \varphi_{\perp}, \nabla_{y} \bar{\psi}\right)_{\Xi_{R}} & =\int_{\mathbb{B}_{R}} \nabla_{y} \bar{\psi}(y) \int_{-h(y)}^{0} \nabla_{y} \varphi_{\perp}(y, z) \mathrm{d} z \mathrm{~d} y \\
& =-\left(\varphi^{-} \nabla_{y} h, \nabla_{y} \bar{\psi}\right)_{\mathbb{B}_{R}} .
\end{aligned}
$$

Similarly,

$$
\left(\left.\varphi_{\perp}\right|_{z=0}, \bar{\psi}\right)_{\mathbb{B}_{R}}=\left(\varphi^{+}, \bar{\psi}\right)_{\mathbb{B}_{R}}
$$

Finally, we prove one more lemma. By virtue of Lemma 2.5 and the relation $\left|\nabla_{y} h(y)\right| \leq c r$, both the expressions (2.43) and (2.44) define continuous functionals in $\bar{\psi} \in V_{2-\beta}^{1}\left(\mathbb{B}_{R}\right)$ and therefore in $\overline{\bar{\psi}} \in V_{1-\beta_{1}}^{1}\left(\mathbb{B}_{R}\right)$, because $1-\beta_{1} \leq 2-\beta$ due to (2.36). Lemma 2.4 yields the same property for $\widehat{F}(\bar{\psi})$. Hence, $\bar{G}(\bar{\psi})$ is a continuous functional in $\bar{\psi} \in V_{1-\beta_{1}}^{1}\left(\mathbb{B}_{R}\right)$, see $(2.40)$. Also

$$
\left((h-H) \nabla_{y} \bar{\varphi}, \nabla_{y} \bar{\psi}\right)_{\mathbb{B}_{R}}
$$

can be regarded as a functional in $\bar{\psi} \in V_{1-\beta_{1}}^{1}\left(\mathbb{B}_{R}\right)$, by taking into account the relation $h(y)-H(y)=\mathcal{O}\left(r^{3}\right)$ (see (1.3)). Finally, we recall that the function $\bar{\varphi}$ vanishes near the circle $\mathbb{S}_{R}=\partial \mathbb{B}_{R}$ and can thus be extended as null from $\mathbb{B}_{R}$ onto $\mathbb{R}^{2}$.

Taking all these remarks into account reduces the identity (2.39) to the integral identity (2.25) of the limit equation (2.26); any test function $u \in V_{-\beta}^{1}\left(\mathbb{R}^{2}\right)$ is allowed, since $\bar{\psi}=\left.u\right|_{\mathbb{B}_{R}} \in V_{-\beta^{1}}\left(\mathbb{R}^{2}\right)$. This proves the following assertion.

Lemma 2.10. Let the indices $\beta$ and $\beta_{1}$ both satisfy (2.27) and in addition $\beta_{1}<\beta \leq \beta_{1}+1 / 2$. Let $F$ be as in (2.36), and assume that $\varphi \in V_{\beta}^{1}(\Omega)$ be as above, solving (1.14) with $\widehat{F}$. Then the functional $\bar{G}$, (2.40), belongs to $V_{1-\beta}^{1}\left(\mathbb{R}^{2}\right)^{*}$; the function $\bar{\varphi}$ belongs to the space $V_{1+\beta}^{1}\left(\mathbb{R}^{2}\right)$ and satisfies the integral identity (2.25) with $G:=\bar{G}$ for all test functions $u \in V_{1-\beta}^{1}\left(\mathbb{R}^{2}\right)$. In particular, $\bar{\varphi}$ has the representation $(2.28-2.30)$ of Theorem 2.2 with

$$
v_{2}(y)=\bar{\varphi}(y)=\frac{1}{h(y)} \int_{-h(y)}^{0} \varphi(y, z) \mathrm{d} z
$$

for some solution $v_{1} \in V_{1+\beta_{1}}^{1}\left(\mathbb{R}^{2}\right)$ of $(2.25)$.

\subsection{The theorem on asymptotics}

We are in the position to conclude the main result of this section. To simplify the presentation we assume in the proof that the solution and the data, in particular the spectal parameter $\lambda$ are real. The complex case holds true as well, see the remark after the proof.

The cut-off function $\chi \in C^{\infty}(\bar{\Omega})$ is as in the beginning of Section 2.6. 
Theorem 2.11. Let the two weight indices $\beta_{1}, \beta_{2}=\beta$ and the functional $F$ satisfy the condition (2.27) and the relations (2.36). Let $\varphi \in V_{\beta}^{1}(\Omega)$ be a solution of the problem (1.14). Then it admits the asymptotic decomposition

$$
\varphi(x)=\chi(x) \sum_{j} c^{(j)} w^{(j)}(y)+\widetilde{\varphi}(x)
$$

where the sum on the right has $2 N_{q}$ terms, and $c^{(j)}$ and $w^{(j)}$ have the same meaning as in (2.28) and (2.29), and the remainder $\widetilde{\varphi}$ belongs to $V_{\beta_{1}}^{1}(\Omega)$. The following estimate is in addition valid:

$$
\left\|\widetilde{\varphi} ; V_{\beta_{1}}^{1}(\Omega)\right\|+\sum_{j}\left|c^{(j)}\right| \leq c\left(\left\|F ; V_{-\beta_{1}}^{1}(\Omega)^{*}\right\|+\left\|\varphi ; V_{\beta}^{1}(\Omega)\right\|\right) .
$$

Proof. Let $\varphi \in V_{\beta}^{1}(\Omega)$ be a solution of the problem (1.14) and $\chi$ as in the assumption. Since $(1-\chi) \varphi \in V_{\beta_{1}}^{1}(\Omega)$, it is enough to prove (2.46) for the function $\chi \varphi$, which we redefine as $\varphi$. By Lemma 2.7, the new $\varphi$ still satisfies equation (1.14) with $\widehat{F}$. We decompose $\varphi=\bar{\varphi}+\varphi_{\perp}$.

By Lemma 2.10, $\bar{\varphi}$ has the representation (2.28):

$$
\bar{\varphi}(x)=\sum_{q} c^{(q)} w^{(q)}(y)+v_{1}(x) .
$$

This can be still multiplied by $\chi$, due to the support of $\bar{\varphi}$. We obtain the first term on the right hand side of (2.46). The rest on the right hand side of (2.48) falls into the term $\widetilde{\varphi}$ in $(2.46)$, since $v_{1} \in V_{1+\beta_{1}}^{1}\left(\mathbb{R}^{2}\right)$ implies that the function $\chi v_{1}$ belongs to $V_{\beta_{1}}^{1}(\Omega)$, as seen by a direct calculation of the norm.

We need to verify the inclusion $\varphi_{\perp} \in V_{\beta_{1}}^{1}(\Omega)$ in order to complete the proof. To this end, we take into account that supp $\varphi_{\perp} \subset \Xi_{R}$ as usual.

Let us introduce the continuous weight function

$$
\mathcal{W}(y)=\left\{\begin{aligned}
r^{\beta_{1}} & \text { for } r>\rho \\
r^{\beta} \rho^{\beta_{1}-\beta} & \text { for } r \leq \rho,
\end{aligned}\right.
$$

where $\rho$ is a positive parameter, to be sent to 0 at the end of the proof. By definition, $\mathcal{W}(y)=\mathcal{O}\left(r^{\beta}\right)$ as $r \rightarrow+0$, but at the limit $\rho \rightarrow+0$, it becomes equal to $r^{\beta_{1}}$. In the sequel we prove that the norms $\left\|\nabla_{x}\left(\mathcal{W} \varphi_{\perp}\right) ; L^{2}\left(\Xi_{R}\right)\right\|$ and $\left\|r^{-2} \mathcal{W} \varphi_{\perp} ; L^{2}\left(\Xi_{R}\right)\right\|$ are finite for any $\rho>0$ so that passing to the limit puts $\varphi_{\perp}$ into the space $V_{\beta_{1}}\left(\Xi_{R}\right)$. Moreover, a crucial trick will just be an application of the Poincaré inequality in the small interval $(-h(y), 0) \ni z$, which is possible due to the orthogonality condition in (1.19).

Clearly,

$$
\left|\nabla_{y} \mathcal{W}(y)\right| \leq c r^{-1} \mathcal{W}(y) .
$$

Furthermore, if $v \in V_{\beta}^{1}\left(\Xi_{R}\right)$, then $\mathcal{W}^{2} v \in V_{-\beta}^{1}(\Omega)$. Hence, we insert $\mathcal{W}^{2} \varphi_{\perp}$ as a test function into (2.41) and obtain

$$
\begin{aligned}
G_{\perp}\left(\mathcal{W}^{2} \varphi_{\perp}\right)= & \left(\nabla_{x} \varphi_{\perp}, \nabla_{x}\left(\mathcal{W}^{2} \varphi_{\perp}\right)\right)_{\Xi_{R}} \\
= & \left(\nabla_{x} \varphi_{\perp}, \mathcal{W} \nabla_{x}\left(\mathcal{W} \varphi_{\perp}\right) \Xi_{\Xi_{R}}+\left(\nabla_{x} \varphi_{\perp}, \mathcal{W} \varphi_{\perp} \nabla_{x} \mathcal{W}\right)_{\Xi_{R}}\right. \\
= & \left\|\nabla_{x}\left(\mathcal{W} \varphi_{\perp}\right) ; L^{2}\left(\Xi_{R}\right)\right\|^{2}-\left\|\varphi_{\perp} \nabla_{x} \mathcal{W} ; L^{2}\left(\Xi_{R}\right)\right\|^{2} \\
& -\left(\varphi_{\perp} \nabla_{x} \mathcal{W}, \nabla_{x}\left(\mathcal{W} \varphi_{\perp}\right)\right)_{\Xi_{R}}+\left(\nabla_{x}\left(\mathcal{W} \varphi_{\perp}\right), \varphi_{\perp} \nabla_{x} \mathcal{W}\right) \Xi_{R}
\end{aligned}
$$


where the last two terms cancel each other due to our assumption on real data. Using the Poincaré inequality (see Lem. 2.6 and $c f$. the calculation (2.35)) we derive the estimate

$$
\left\|\nabla_{x}\left(\mathcal{W} \varphi_{\perp}\right) ; L^{2}\left(\Xi_{R}\right)\right\|^{2} \geq c\left\|h^{-1} \mathcal{W} \varphi_{\perp} ; L^{2}\left(\Xi_{R}\right)\right\|^{2} .
$$

Thus, fixing $R$ small enough, the formulas (2.50) and (1.3), (1.4) imply $\operatorname{ch}(y)^{-1} \mathcal{W}(y) \geq 2\left|\nabla_{y} \mathcal{W}(y)\right|$ so that the right hand side of (2.51) is bigger than

$$
c\left\|\mathcal{W} \varphi_{\perp} ; V_{0}^{1}\left(\Xi_{R}\right)\right\|^{2}=c\left\|\nabla_{x}\left(\mathcal{W} \varphi_{\perp}\right) ; L^{2}\left(\Xi_{R}\right)\right\|^{2}+c\left\|r^{-1} \mathcal{W} \varphi_{\perp} ; L^{2}\left(\Xi_{R}\right)\right\|^{2}, c>0 .
$$

Let us consider the left hand side of (2.51): we estimate the terms in (2.42). First, we recall that $\widehat{F} \in$ $V_{-\beta_{1}}^{1}(\Omega)^{*}$ and $r^{-\beta_{1}} \mathcal{W}(y) \leq 1$ due to $(2.49)$ so that

$$
\begin{aligned}
\left|\widehat{F}\left(\mathcal{W}^{2} \varphi_{\perp}\right)\right| & \leq c_{F}\left\|\mathcal{W}^{2} \varphi_{\perp} ; V_{-\beta_{1}}^{1}(\Omega)\right\|^{2} \\
& =c_{F}\left(\int_{\Xi_{R}} r^{-2 \beta_{1}}\left(\left|\nabla_{x}\left(\mathcal{W}(y)^{2} \varphi_{\perp}(x)\right)\right|^{2}+r^{-2} \mathcal{W}(y)^{4}\left|\varphi_{\perp}(x)\right|^{2}\right) \mathrm{d} x\right)^{1 / 2} \\
& \leq c\left(\left\|\nabla_{x}\left(\mathcal{W} \varphi_{\perp}\right) ; L^{2}\left(\Xi_{R}\right)\right\|^{2}+\left\|r^{-1} \mathcal{W} \varphi_{\perp} ; L^{2}\left(\Xi_{R}\right)\right\|^{2}\right)^{1 / 2}=c\left\|\mathcal{W} \varphi_{\perp} ; V_{0}^{1}\left(\Xi_{R}\right)\right\| .
\end{aligned}
$$

Second, by the calculation (2.43) with $\varphi_{\perp}, \bar{\psi}$ changed for $\mathcal{W} \varphi_{\perp}, \bar{\varphi}$, we have

$$
\begin{aligned}
\left|\left(\nabla_{y} \bar{\varphi}, \nabla_{y}\left(\mathcal{W}^{2} \varphi_{\perp}\right)\right)_{\Xi_{R}}\right| & =\left|\left(\nabla_{y} \bar{\varphi}, \nabla_{y} h \mathcal{W}^{2} \varphi^{-}\right)_{\mathbb{B}_{R}}\right| \\
& \leq c \max _{y \in \mathbb{B}_{R}}\left(r^{1-2 \beta} \mathcal{W}(y)^{2}\right)\left\|r^{\beta+1} \nabla_{y} \bar{\varphi} ; L^{2}\left(\mathbb{B}_{R}\right)\right\|\left\|r^{\beta-1} \varphi^{-} ; L^{2}\left(\mathbb{B}_{R}\right)\right\| .
\end{aligned}
$$

Here we have taken into account Lemmas 2.4 and 2.5. Third, referring to Lemmas 1.1 and 2.5, we obtain

$$
\left|\left(\left.\varphi\right|_{z=0},\left.\mathcal{W}^{2} \varphi_{\perp}\right|_{z=0}\right)_{\mathbb{B}_{R}}\right| \leq c \max _{y \in \mathbb{B}_{R}}\left(r^{1-2 \beta} \mathcal{W}(y)^{2}\right)\left\|\left.r^{\beta} \varphi\right|_{z=0} ; L^{2}\left(\mathbb{B}_{R}\right)\right\|\left\|r^{\beta-1} \varphi^{+} ; L^{2}\left(\mathbb{B}_{R}\right)\right\| .
$$

Since $1-2 \beta+2 \beta_{1} \geq 0$ and $\mathcal{W}(y) \leq r^{\beta_{1}}$ (see (2.36) and (2.49)), both maxima in (2.52) and (2.53) exist. Moreover, the products of the norms do not exceed $c\left\|\varphi ; V_{\beta}^{1}\left(\Xi_{R}\right)\right\|^{2}$. Hence, the relation (2.51) implies

$$
c_{1}\left\|\widehat{F} ; V_{-\beta_{1}}^{1}\left(\Xi_{R}\right)^{*}\right\|\left\|\mathcal{W} \varphi_{\perp} ; V_{0}^{1}\left(\Xi_{R}\right)\right\|+c_{2}\left\|\varphi ; V_{\beta}^{1}\left(\Xi_{R}\right)\right\|^{2} \geq\left\|\mathcal{W} \varphi_{\perp} ; V_{0}^{1}\left(\Xi_{R}\right)\right\|^{2}
$$

and

$$
\left\|\mathcal{W} \varphi_{\perp} ; V_{0}^{1}\left(\Xi_{R}\right)\right\| \leq c\left(\left\|\varphi ; V_{\beta}^{1}\left(\Xi_{R}\right)\right\|+\left\|\widehat{F} ; V_{-\beta_{1}}^{1}\left(\Xi_{R}\right)^{*}\right\|\right)
$$

Keeping in mind the monotonicity of the weight function and passing to the limit $\rho \rightarrow+0$ in (2.54) yield

$$
\left\|r^{\beta_{1}} \varphi_{\perp} ; V_{0}^{1}\left(\Xi_{R}\right)\right\| \leq c\left(\left\|\varphi ; V_{\beta}^{1}\left(\Xi_{R}\right)+\right\| F ; V_{-\beta_{1}}^{1}\left(\Xi_{R}\right)^{*} \|\right) .
$$

The norm on the left is equivalent to $\left\|\varphi_{\perp} ; V_{\beta_{1}}^{1}\left(\Xi_{R}\right)\right\|$. Theorem 2.11 is proven.

In the case of complex data it is enough only to extract the real part of all terms in (2.51); then the last two terms again disappear. Otherwise the proof is the same. 


\section{The Fredholm property of $A_{\beta}(\lambda)$}

In this chapter we present a necessary and sufficient condition of the Fredholmess of the operator $A_{\beta}(\lambda)$, see Theorem 3.2. In the last section we also compute its index. This will be closely connected to the number of the power law solutions of the limit equation, which was studied in the preceding chapter.

\subsection{Estimating the solution}

The purpose of this section is just to derive the following estimate (3.1), which will be needed in the proof of Theorem 3.2.

Lemma 3.1. Assume that $\beta$ meets the conditions $(2.27)$ and let $\varphi \in V_{\beta}^{1}(\Omega)$ be a solution of the problem (1.14). It satisfies

$$
\left\|\varphi ; V_{\beta}^{1}(\Omega)\right\| \leq c_{\beta}\left(\left\|F ; V_{-\beta}^{1}(\Omega)^{*}\right\|+\left\|\varphi ; L_{\beta-1 / 2}^{2}(\Omega)\right\|\right)
$$

Proof. We first mention the following local elliptic estimate (see, e.g. $[16,17])$

$$
\left\|\varphi ; H^{1}\left(\Omega \backslash \Xi_{R / 2}\right)\right\| \leq c\left(\left\|F ; V_{-\beta}^{1}(\Omega)^{*}\right\|+\left\|\varphi ; L^{2}\left(\Omega \backslash \Xi_{R / 4}\right)\right\|\right) .
$$

Note that since $\varrho \geq c>0$ on $\Omega \backslash \Xi_{R / 4}$, the first term on the right is a majorant for the norm of the functional

$$
H^{1}\left(\Omega \backslash \Xi_{R / 4}\right) \ni u \mapsto F\left(\left(1-\chi^{\prime}\right) u\right),
$$

where $\chi^{\prime}$ is a cut-off function, which equals to one in $\Xi_{R / 4}$ and vanishes in $\Omega \backslash \Xi_{R / 2}$. Using the same argument with the weight $\varrho$, we rewrite $(3.2)$ as follows:

$$
\left\|\varphi ; V_{\beta}^{1}\left(\Omega \backslash \Xi_{R / 2}\right)\right\| \leq c\left(\left\|F ; V_{-\beta}^{1}(\Omega)^{*}\right\|+\left\|\varphi ; L_{\beta-1 / 2}^{2}(\Omega)\right\|\right) .
$$

Moreover, in the blanket we consider the product $\widehat{\varphi}=\chi^{\prime} \varphi$ satisfying (1.14) with the new right hand side $\widehat{F}$ (cf. Lem. 2.7),

$$
\left\|\widehat{F} ; V_{-\beta}^{1}\left(\Xi_{R}\right)^{*}\right\| \leq c\left(\left\|F ; V_{-\beta}^{1}\left(\Xi_{R}\right)^{*}\right\|+\left\|\varphi ; V_{\beta}^{1}\left(\Omega \backslash \Xi_{R / 2}\right)\right\|\right)
$$

It is plain that $\hat{F}$ and its norm $\left\|\widehat{F} ; V_{-\beta}^{1}\left(\Xi_{R}\right)^{*}\right\|$ depend on $R$, however, all constants in the estimates below can be chosen independent of $R$ : this is evident in Lemmas 2.4-2.6 while the Kondratiev theorem on asymptotics (Thm. 2.11) is applied in the whole plane $\mathbb{R}^{2}$.

In the following we cease to display the hat for $\widehat{\varphi}$ and consider the problems (2.39) and (2.41) for the terms $\bar{\varphi}$ and $\varphi_{\perp}$ of the decomposition (1.18). We use the notation of Section 2.6 for $\bar{G}$, etc. and modify the arguments of that section as follows.

First, we extend $\bar{\varphi}$ as zero from $\mathbb{B}_{R}$ onto $\mathbb{R}^{2}$. As in the proof of Lemma 2.10 we see that $\bar{\varphi}$ satisfies the limit equation (2.25) with the new right hand side functional $u \mapsto \bar{G}(u)+\left((h-H) \nabla_{y} \bar{\varphi}, \nabla_{y} u\right)_{\mathbb{B}_{R}}$, and this leads to the bound

$$
\left\|\bar{\varphi} ; V_{1+\beta}^{1}\left(\mathbb{B}_{R}\right)\right\| \leq c\left(\left\|\bar{G} ; V_{1-\beta}^{1}\left(\mathbb{B}_{R}\right)^{*}\right\|+\left\|(h-H) \nabla_{y} \bar{\varphi} ; L_{\beta-1}^{2}\left(\mathbb{B}_{R}\right)\right\|\right) .
$$

Processing the right hand side, we have

$$
\begin{aligned}
\left\|(h-H) \nabla_{y} \bar{\varphi} ; L_{\beta-1}^{2}\left(\mathbb{B}_{R}\right)\right\| & \leq c\left\|r^{3} \nabla_{y} \bar{\varphi} ; L_{\beta-1}^{2}\left(\mathbb{B}_{R}\right)\right\| \leq c\left\|\nabla_{y} \bar{\varphi} ; L_{\beta+2}^{2}\left(\mathbb{B}_{R}\right)\right\| \\
& \leq c\left\|\bar{\varphi} ; V_{\beta+2}^{1}\left(\mathbb{B}_{R}\right)\right\| \leq c\left\|\varphi ; V_{\beta+1}^{1}\left(\Xi_{R}\right)\right\| .
\end{aligned}
$$


Here we used Lemma 2.4 with $\beta$ replaced by $\beta+1$. The same substitution in Lemma 2.5 , and (2.43), (2.44) yield the following inequalities for the last two terms in (2.40):

$$
\begin{aligned}
\left|\left(\nabla_{y} \bar{\varphi}, \nabla_{y} \psi_{\perp}\right)_{\Xi_{R}}\right| & =\left|\left(\varphi^{-} \nabla_{y} h, \nabla_{y} \bar{\psi}\right)_{\mathbb{B}_{R}}\right| \\
& \leq c\left\|r^{\beta} \varphi^{-} ; L^{2}\left(\mathbb{B}_{R}\right)\right\|\left\|r^{1-\beta} \nabla_{y} \bar{\psi} ; L^{2}\left(\mathbb{B}_{R}\right)\right\| \\
& \leq c\left\|\varphi ; V_{\beta+1}^{1}\left(\Xi_{R}\right)\right\|\left\|\bar{\psi} ; V_{1-\beta}^{1}\left(\mathbb{B}_{R}\right)\right\|, \\
\left|\left(\left.\varphi_{\perp}\right|_{z=0}, \bar{\psi}\right)_{\mathbb{B}_{R}}\right| & \leq c\left\|r^{\beta} \varphi^{+} ; L^{2}\left(\mathbb{B}_{R}\right)\right\|\left\|r^{-\beta} \bar{\psi} ; L^{2}\left(\mathbb{B}_{R}\right)\right\| \\
& \leq c\left\|\varphi ; V_{\beta+1}^{1}\left(\Xi_{R}\right)\right\|\left\|\bar{\psi} ; V_{1-\beta}^{1}\left(\mathbb{B}_{R}\right)\right\| .
\end{aligned}
$$

In other words, we have

$$
\begin{aligned}
\left\|\bar{\varphi} ; V_{\beta}^{1}\left(\Xi_{R}\right)\right\| & \leq c\left\|\bar{\varphi} ; V_{\beta+1}^{1}\left(\mathbb{B}_{R}\right)\right\| \\
& \leq c\left(\left\|F ; V_{-\beta}^{1}\left(\Xi_{R}\right)^{*}\right\|+\left\|\varphi ; V_{\beta+1}^{1}\left(\Xi_{R}\right)\right\|\right) .
\end{aligned}
$$

The estimate (2.55) for the component $\varphi_{\perp}$ holds for any weight indices $\beta_{1}$ and $\beta_{2}=\beta$ such that $\beta_{1} \in$ $\left[\beta_{2}-1 / 2, \beta_{2}\right)(c f .(2.36))$. Taking $\beta_{1}=\beta$ and $\beta_{2}=\beta+1 / 2$ we thus obtain

$$
\left\|\varphi_{\perp} ; V_{\beta}^{1}\left(\Xi_{R}\right)\right\| \leq c\left(\left\|F ; V_{-\beta}^{1}\left(\Xi_{R}\right)^{*}\right\|+\left\|\varphi ; V_{\beta+1 / 2}^{1}\left(\Xi_{R}\right)\right\|\right) .
$$

Combining the inequalities (3.3), (3.4), (3.6), and (3.7) yields

$$
\begin{aligned}
\left\|\varphi ; V_{\beta}^{1}(\Omega)\right\| \leq & c\left(\left\|\varphi ; V_{\beta}^{1}\left(\Omega \backslash \Xi_{R / 2}\right)\right\|+\left\|\bar{\varphi} ; V_{\beta}^{1}\left(\Xi_{R}\right)\right\|+\left\|\varphi_{\perp} ; V_{\beta}^{1}\left(\Xi_{R}\right)\right\|\right) \\
\leq & C\left\|F ; V_{-\beta}^{1}\left(\Xi_{R}\right)^{*}\right\|+C\left\|\varphi ; L_{\beta-1 / 2}^{2}(\Omega)\right\| \\
& +C\left\|\varphi ; V_{\beta+1}^{1}\left(\Xi_{R}\right)\right\|+C\left\|\varphi ; V_{\beta+1 / 2}^{1}\left(\Xi_{R}\right)\right\| .
\end{aligned}
$$

Recalling the definition of the weighted Kondratiev norm (1.10) we get the estimate

$$
\left\|\varphi ; V_{\beta+1 / 2}^{1}\left(\Xi_{R}\right)\right\| \leq\left\|\varphi ; V_{\beta}^{1}\left(\Xi_{R}\right)\right\| \sup _{x \in \Xi_{R}} \varrho(x)^{1 / 2} \leq\left\|\varphi ; V_{\beta}^{1}(\Omega)\right\| \sup _{x \in \Xi_{R}} \varrho(x)^{1 / 2}
$$

and similarly for $\left\|\varphi ; V_{\beta+1}^{1}\left(\Xi_{R}\right)\right\|$. This means, taking a small enough $R>0$, the last two terms on the right hand side of (3.8) can be bounded by a small constant times $\left\|\varphi ; V_{\beta}^{1}(\Omega)\right\|$. Moving these to the left hand side, the desired estimate (3.1) follows.

\subsection{The Fredholm property}

Everything is prepared for checking the following assertion for the operator $A_{\beta}(\lambda): V_{\beta}^{1}(\Omega) \rightarrow V_{-\beta}^{1}(\Omega)^{*}$, defined in (1.15).

Theorem 3.2. Let the weight index $\beta \in \mathbb{R}$ be given. The operator $A_{\beta}(\lambda): V_{\beta}^{1}(\Omega) \rightarrow V_{-\beta}^{1}(\Omega)^{*}$ is Fredholm, if and only if every $\Lambda_{j}^{ \pm}, j \in \mathbb{N}$ (defined in (2.17-2.19), satisfies (2.27), i.e. $\operatorname{Re} \Lambda_{j}^{ \pm} \neq-1-\beta$.

In case $\operatorname{Re} \Lambda_{j}^{ \pm}=-1-\beta$ the range $\operatorname{Im} A_{\beta}(\lambda)$ is not a closed subspace in $V_{-\beta}^{1}(\Omega)^{*}$. 
Proof. Since the embedding $V_{\beta}^{1}(\Omega) \subset L_{\beta-1 / 2}^{2}(\Omega)$ is compact, the estimate (3.1) ensures that the kernel of $A_{\beta}(\lambda)$ is finite dimensional and the range is closed. This is well known, see e.g. Lemma 3 of [39]. Since $A_{\beta}(\lambda)^{*}=A_{-\beta}(\lambda)$ and since the estimate (3.1) also holds for $-\beta$ instead of $\beta$ (see Rem. 2.3), the co-kernel

$$
\operatorname{coker} A_{\beta}(\lambda)=\operatorname{ker} A_{-\beta}(\lambda)
$$

is also finite-dimensional. Hence, $A_{\beta}(\lambda)$ is Fredholm.

Let us present the idea how to prove the last assertion of the theorem. First, since $V_{\beta}^{1}(\omega) \subset V_{\beta+\delta}^{1}(\Omega)$ and since a small $\delta>0$ can be found such that the condition (2.27) is met by $\beta+\delta$ and $A_{\beta+\delta}(\lambda)$ is Fredholm, we obtain

$$
\operatorname{ker} A_{\beta}(\lambda) \subset \operatorname{ker} A_{\beta+\delta}(\lambda) \text { and } \operatorname{dim} \operatorname{ker} A_{\beta}(\lambda)<\infty .
$$

Second, the closedness of the range $\operatorname{Im} A_{\beta}(\lambda)$ guarantees the inequality

$$
\left\|\varphi ; V_{\beta}^{1}(\Omega)\right\| \leq c\left\|A_{\beta}(\lambda) \varphi ; V_{-\beta}^{1}(\Omega)^{*}\right\|, \varphi \in V_{\beta}^{1}(\Omega) \ominus \operatorname{ker} A_{\beta}(\lambda),
$$

but it will not be valid for suitably chosen functions $v_{m}$ (to be defined), for sufficiently large $m$ (compare (3.24) and (3.15)). Third, we observe that

$$
\operatorname{supp} v^{m} \cap \operatorname{supp} v^{q}=\varnothing \text { for } m \neq q,
$$

and therefore these functions cannot be linearly independent. These three facts together ensure that the range $\operatorname{Im} A_{\beta}(\lambda)$ is not closed, completing the proof of the theorem.

To present the details, we consider a nontrivial power-law solution (1.20) with the exponent $\Lambda$ such that

$$
\operatorname{Re} \Lambda=-1-\beta
$$

$(c f .(2.27))$ and the family of functions

$$
\widehat{v}^{m}(y)=X_{m}(r) v(y), m \in \mathbb{N},
$$

where $X_{m}$ is a plateau function defined by

$$
X_{m}(r)=\chi_{0}\left(2^{m}+\ln \frac{r}{R}\right) \chi_{0}\left(-2^{m+1}-\ln \frac{r}{R}\right)
$$

and $\chi_{0}$ is a standard cut-off function, $\chi_{0}(t)=1$ for $t \leq 0$ and $\chi_{0}(t)=0$ for $t \geq 1$. Since the function (3.14) is equal to one in the segment

$$
\Upsilon_{m}=\left[R \exp \left(-2^{m+1}\right), R \exp \left(-2^{m}\right)\right] \ni r,
$$

we have

$$
\begin{aligned}
\left\|\widehat{v}^{m} ; V_{\beta}^{1}(\Omega)\right\|^{2} & \geq\left\|\widehat{v}^{m} ; L_{\beta-1}^{2}(\Omega)\right\|^{2} \\
& =\int_{\mathbb{S}^{1}} \int_{0}^{R} \int_{-h(y)}^{0} r^{2(\beta-1)} X_{m}(r)^{2}|v(y)|^{2} \mathrm{~d} z r \mathrm{~d} r \mathrm{~d} \vartheta \\
& \geq c_{v} \int_{\Upsilon_{m}} r^{2(\beta-1)} r^{2 \operatorname{Re} \Lambda} r \mathrm{~d} r=c_{v} \int_{\Upsilon_{m}} \frac{\mathrm{d} r}{r}=c_{v}\left(2^{m+1}-2^{m}\right) .
\end{aligned}
$$


Hence, the normalized function $v^{m}:=2^{-m / 2} \widehat{v}^{m}$ satisfies

$$
\left\|v^{m} ; V_{\beta}^{1}(\Omega)\right\| \geq C>0
$$

and a similar calculation shows that

$$
\left\|v^{m} ; V_{\beta}^{1}(\Omega)\right\| \leq\left\|v^{m} ; V_{\beta+1}^{2}(\Omega)\right\| \leq C^{\prime}
$$

for some positive constants $C, C^{\prime}$.

Let us compute the norm

$$
\left\|A_{\beta}(\lambda) v^{m} ; V_{-\beta}^{1}(\Omega)^{*}\right\|=\sup \left|\left(\nabla_{x} v^{m}, \nabla_{x} u\right)_{\Omega}-\lambda\left(v^{m}, u\right)_{\Gamma}\right|
$$

where the supremum is taken over all $u \in V_{-\beta}^{1}(\Omega)$ such that $\left\|u ; V_{-\beta}^{1}(\Omega)\right\|=1$. Since $v^{m}=0$ outside the blanket $\Xi_{R / 2}$, we can make the restriction stronger, i.e., supp $u \subset \overline{\Xi_{R}}$. We then employ the decomposition $u=\bar{u}+u_{\perp}$ as in (1.18) and (1.19). Integrating by parts, we deduce that

$$
\begin{aligned}
\left(\nabla_{x} v^{m}, \nabla_{x} u\right)_{\Omega}-\lambda\left(v^{m}, u\right)_{\Gamma}= & \left(-\Delta_{x} v^{m}, u\right)_{\Omega}-\left(\partial_{z} v^{m}-\lambda v^{m}, u\right)_{\Gamma}+\left(\partial_{\nu} v^{m}, u\right)_{\partial \Theta} \\
= & \left(-h \Delta_{x} v^{m}-\nabla_{y} h \cdot \nabla_{y} v^{m}-\lambda v^{m}, \bar{u}\right)_{\mathbb{B}_{R}} \\
& +\left(-\Delta_{x} v^{m}, u_{\perp}\right)_{\Xi_{R}}-\lambda\left(v^{m}, u_{\perp}\right)_{\Gamma \cap \partial \Xi_{R}}+\left(\partial_{\nu} v^{m}, u\right)_{\partial \Theta \cap \partial \Xi_{R}} .
\end{aligned}
$$

By Lemmas 2.6 and 2.5, and the estimate (3.16), we have

$$
\begin{aligned}
\left|\left(\Delta_{x} v^{m}, u_{\perp}\right) \Xi\right| & \leq c\left\|v^{m} ; V_{\beta+1}^{2}\left(\Xi_{R_{m}}\right)\right\|\left\|u_{\perp} ; L_{-\beta-1}^{2}\left(\Xi_{R_{m}}\right)\right\| \\
& \leq c\left\|v^{m} ; V_{\beta+1}^{2}(\Omega)\right\| R_{m}\left\|u_{\perp} ; L_{-\beta-2}^{2}\left(\Xi_{R_{m}}\right)\right\| \\
& \leq c \mathbf{C} R_{m}\left\|u ; V_{-\beta}^{1}(\Omega)\right\|, \\
\left|\left(v^{m}, u_{\perp}\right)_{\Gamma \cap \partial \Xi_{R}}\right| & \leq c\left\|v^{m} ; L_{\beta}^{2}\left(\partial \Xi_{R_{m}} \cap \Gamma\right)\right\|\left\|u_{\perp} ; L_{-\beta}^{2}\left(\partial \Xi_{R_{m}} \cap \Gamma\right)\right\| \\
& \leq c\left\|v^{m} ; V_{\beta}^{1}\left(\Xi_{R}\right)\right\| R_{m}\left\|u_{\perp} ; L_{-\beta-1}^{2}\left(\partial \Xi_{R_{m}} \cap \Gamma\right)\right\| \\
& \leq c \mathbf{C} R_{m}\left\|u_{\perp} ; V_{-\beta}^{1}(\Omega)\right\| .
\end{aligned}
$$

Here $R_{m}=R \exp \left(-2^{m}+1\right)$ is fixed such that $\operatorname{supp} v^{m} \subset \overline{\Xi_{R_{m}}}$. Furthermore, $\partial_{\nu} v^{m}(y)=-\left(1+\left|\nabla_{y} h(y)\right|^{2}\right)^{-1 / 2}$ $\nabla_{y} h(y) \cdot \nabla_{y} v^{m}(y)(c f .(1.2-1.4)$, and so

$$
\begin{aligned}
\mid\left(\partial_{\nu} v^{m}, u\right)_{\partial \Theta \cap \partial \Xi_{R} \mid} & \leq c\left\|r \nabla_{y} v^{m} ; L_{\beta}^{2}\left(\partial \Theta \cap \partial \Xi_{R_{m}}\right)\right\|\left\|u_{\perp} ; L_{-\beta}^{2}\left(\partial \Theta \cap \partial \Xi_{R_{m}}\right)\right\| \\
& \leq c\left\|\nabla_{y} v^{m} ; L_{\beta+1}^{2}\left(\partial \Theta \cap \partial \Xi_{R}\right)\right\| R_{m}\left\|u_{\perp} ; L_{-\beta-1}^{2}\left(\partial \Theta \cap \partial \Xi_{R}\right)\right\| \\
& \leq c\left\|v^{m} ; V_{\beta+1}^{2}\left(\Xi_{R}\right)\right\| R_{m}\left\|u_{\perp} ; V_{-\beta}^{1}\left(\Xi_{R}\right)\right\| \\
& \leq c \mathbf{C} R_{m}\left\|u_{\perp} ; V_{-\beta}^{1}\left(\Xi_{R}\right)\right\| .
\end{aligned}
$$

Notice that $R_{m} \rightarrow 0$ as $m \rightarrow 0$. 
Based on Lemma 2.4 and formula (1.3), we change $h$ for $H$ in the first scalar product on the right of (3.18) and observe that

$$
\begin{aligned}
\left|\left((h-H) \Delta_{y} v^{m}+\nabla_{y}(h-H) \cdot \nabla_{y} v^{m}, \bar{u}\right)_{\mathbb{B}_{R}}\right| \leq & c\left(\left\|r^{\beta+3} \Delta_{y} v^{m} ; L^{2}\left(\mathbb{B}_{R_{m}}\right)\right\|\right. \\
& \left.+\left\|r^{\beta+2} \nabla_{y} v^{m} ; L^{2}\left(\mathbb{B}_{R_{m}}\right)\right\|\right)\left\|\bar{u} ; L_{-\beta}^{2}\left(\mathbb{B}_{R_{m}}\right)\right\| \\
\leq & c R_{m}\left(\left\|r^{\beta+1} \nabla_{x}^{2} v^{m} ; L^{2}\left(\Xi_{R_{m}}\right)\right\|\right. \\
& \left.+\left\|r^{\beta} \nabla_{x} v^{m} ; L^{2}\left(\mathbb{B}_{R_{m}}\right)\right\|\right)\left\|\bar{u} ; L_{-\beta+1}^{2}\left(\mathbb{B}_{R_{m}}\right)\right\| \\
\leq & c R_{m}\left\|v^{m} ; V_{\beta+1}^{2}\left(\Xi_{R}\right)\right\|\left\|u ; V_{-\beta}^{1}\left(\Xi_{R}\right)\right\| .
\end{aligned}
$$

Furthermore, since $v$ is a power-law solution of the limit equation, the expression

$$
-H(y) \Delta_{y} v^{m}(y)-\nabla_{y} H(y) \cdot \nabla_{y} v^{m}(y)-\lambda v^{m}(y)=-\nabla_{y} \cdot H(y) \nabla_{y} v^{m}(y)-\lambda v^{m}(y)
$$

is nonzero only in the case

$$
\begin{aligned}
r \in \Upsilon_{m}^{\prime}= & \left(R \exp \left(-2^{m+1}-1\right), R \exp \left(-2^{m+1}\right)\right) \\
& \cup\left(R \exp \left(-2^{m}\right), R \exp \left(-2^{m}+1\right)\right)
\end{aligned}
$$

and the modulus of this expression does not exceed

$$
c 2^{-m / 2} r^{\operatorname{Re} \Lambda}
$$

Hence,

$$
\begin{aligned}
\left|\left(\nabla_{y} \cdot H \nabla_{y} v^{m}-\lambda v^{m}, \bar{u}\right)_{\mathbb{B}_{R}}\right| & \leq c 2^{-m / 2} \int_{\Upsilon_{m}^{\prime}} r^{2 \beta} r^{2 \operatorname{Re} \Lambda} r \mathrm{~d} r\left\|r^{-\beta} \bar{u} ; L^{2}\left(\mathbb{B}_{R}\right)\right\| \\
& \leq c 2^{-m / 2}\left\|u ; V_{-\beta}^{1}\left(\Xi_{R}\right)\right\|,
\end{aligned}
$$

because

$$
\int_{R \exp \left(-2^{m}\right)}^{R \exp \left(-2^{m}+1\right)} \frac{\mathrm{d} r}{r}=\ln \left(R \exp \left(-2^{m}+1\right)\right)-\ln \left(R \exp \left(-2^{m}\right)\right)=1
$$

and the same is valid for the integral over the second interval in (3.22).

Collecting the estimates (3.19-3.23) and recalling (3.18), (3.17), we see that

$$
\left\|A_{\beta}(\lambda) v^{m} ; V_{\beta}^{1}(\Omega)^{*}\right\| \leq c 2^{-m / 2} .
$$

The theorem is thus proven.

\subsection{Computing the index}

In the case $\lambda \in\left[0, \lambda_{\dagger}\right)$, the self-adjoint operator $A_{0}(\lambda)$ is Fredholm and therefore

$$
\operatorname{Ind} A_{0}(\lambda)=0
$$


Under the assumption $\lambda \geq \lambda_{\dagger}$ the Fredholm property does no more hold. However, we can fix a number $\delta(\lambda)>0$ such that all exponents of the power-law solutions in the strip

$$
\{\Lambda \in \mathbb{C}:|\operatorname{Re} \lambda+1|<\delta(\lambda)\}
$$

lie on the line

$$
\{\Lambda \in \mathbb{C}: \operatorname{Re} \Lambda=-1\} .
$$

Hence, for $\beta \in(0, \delta(\lambda))$ both the operators $A_{\beta}(\lambda)$ and $A_{-\beta}(\lambda)$ are Fredholm. Since $A_{\beta}(\lambda)=A_{-\beta}(\lambda)^{*}$, we infer using (1.16) and (1.17) that

$$
\operatorname{Ind} A_{\beta}(\lambda)=-\operatorname{Ind} A_{-\beta}(\lambda) .
$$

We apply Theorem 2.11 with $\beta_{1}=-\beta$. To satisfy the condition (2.36), i.e., $\beta \leq-\beta+1 / 2$, we assume in addition that $\delta(\lambda) \leq 1 / 4$ in (3.26). Let $\lambda \in\left[\lambda_{q-1}^{\dagger}, \lambda_{q}^{\dagger}\right)$ for some $q \in \mathbb{N}$ and thresholds taken from (2.13). Then there exist exactly $2 N_{q}$ power-law and power-logarithmic solutions (1.20) and (2.20) with exponents $\Lambda$ in (3.27). Taking any inverse of the mapping $V_{\beta}^{1}(\Omega) \rightarrow \operatorname{Im} A_{\beta}(\lambda)$, we deduce using Theorem 2.11 that the pre-image

$$
\mathbf{V}_{\beta}(\lambda)=A_{\beta}(\lambda)^{-1} V_{\beta}^{1}(\Omega)^{*} \subset V_{\beta}^{1}(\Omega)
$$

of $A_{\beta}(\lambda)$ differs from $V_{-\beta}^{1}(\Omega)$ by $\mathbf{L}$, which is the linear hull of the above mentioned special solutions multiplied by the cut-off funtion $\chi$. In other words, the quotient space

$$
\mathbf{W}(\lambda)=\mathbf{V}_{\beta}(\lambda) / V_{-\beta}^{1}(\Omega)
$$

can be identified with $\mathbb{C}^{2 N_{q}}$. By (2.47), the space $\mathbf{V}_{\beta}(\lambda)$ has the intrinsic (Hilbert space) norm

$$
\left(\left\|\tilde{\varphi} ; V_{\beta_{1}}^{1}(\Omega)\right\|^{2}+\sum_{j}\left|c^{(j)}\right|^{2}\right)^{1 / 2}
$$

for a function $\varphi$ with the representation (2.46). Hence, the quotient (3.30) induces a specific topology in (3.29) so that the restriction $\mathbf{A}_{\beta}(\lambda)$ of $A_{\beta}(\lambda)$ onto $\mathbf{V}_{\beta}(\lambda)$ inherits all general properties, in particular,

$$
\operatorname{Ind} A_{\beta}(\lambda)=\operatorname{Ind} \mathbf{A}_{\beta}(\lambda) .
$$

Owing to $\operatorname{dim} \mathbf{W}(\lambda)=2 N_{q}$, we further have

$$
\operatorname{Ind} A_{\beta}(\lambda)=2 N_{q}+\operatorname{Ind} A_{-\beta}(\lambda) .
$$

Comparing (3.28) and (3.33) we can conlude with the last assertion of this section:

Theorem 3.3. For any $\lambda \geq 0$ there exists $\delta(\lambda) \in(0,1 / 4]$ such that in the case $\lambda \in\left[0, \lambda_{\dagger}\right)$ the operator $A_{\beta}(\lambda)$ with $\beta \in(-\delta(\lambda), \delta(\lambda))$ is of index zero and in the case $\lambda \in\left[\lambda_{q-1}^{\dagger}, \lambda_{q}^{\dagger}\right)$ the operators $A_{\beta}(\lambda)$ and $A_{-\beta}(\lambda)$ with $\beta \in(0, \delta(\lambda))$ satisfy

$$
\operatorname{Ind} A_{\beta}(\lambda)=-\operatorname{Ind} A_{-\beta}(\lambda)=N_{q}
$$

The numbers $\lambda_{\dagger}=\lambda_{1}^{\dagger}$ and $\lambda_{q}^{\dagger}, N_{q}$, where $q \in \mathbb{N}$, are as in Section 2.2. 
We note that Theorem 2.11 passes the equality Ind $A_{0}(\lambda)=0$, observed for $\lambda<\lambda_{\dagger}$, to all operators $A_{\beta}(\lambda)$ with $\beta$ such that the segment $[-1-|\beta|,-1+|\beta|]$ is free of the exponents $\Lambda_{j}^{ \pm}$in $(2.17)$. Since $V_{\beta_{1}}^{1}(\Omega) \subset V_{\beta_{2}}^{1}(\omega)$ for $\beta_{2} \geq \beta_{1}$, the indices of the Fredholm operators $A_{\beta_{1}}(\lambda)$ and $A_{\beta_{2}}(\lambda)$ are related by

$$
\text { Ind } A_{\beta_{1}}(\lambda) \leq \text { Ind } A_{\beta_{2}}(\lambda) \text {. }
$$

Hence, the function

$$
\mathbb{R} \ni \beta \mapsto \operatorname{Ind} A_{\beta}(\lambda)
$$

is piecewise constant monotone increasing. Using Theorem 2.11 iteratively, one may readily express the function (3.35) in terms of the eigenvalues (2.15) of the problem (2.11).

The formula (3.34) shows that in the case $\lambda \geq \lambda_{\dagger}$ the operator $A_{\beta}(\lambda)$ is never of index zero.

\section{RADiATION CONDITIONS AND WAVE PHENOMENON IN THE WATER BLANKET}

In this chapter we continue the study of the quotient space $\mathbf{W}(\lambda)$ of (3.30), which we call the space of blanket waves, and its elements the water waves localized in the water blanket, or shortly the blanket waves. We shall decompose $\mathbf{W}(\lambda)$ into two components $\mathbf{W}^{+}(\lambda)$ and $\mathbf{W}^{-}(\lambda)$ of outgoing and incoming waves. It will turn out that the restriction of the operator $A_{\beta}(\lambda)$ to the direct sum $V_{-\beta}^{1}(\Omega) \oplus \mathbf{W}^{+}(\lambda)$ (rather than the operator on the entire space $\left.V_{-\beta}^{1}(\Omega) \oplus \mathbf{W}(\lambda)\right)$ will be Fredholm of index zero. This observation will lead to the radiation conditions.

\subsection{Waves localized in the water blanket}

In Sections 4.1 and 4.2 we form a basis of $\mathbf{W}(\lambda)$, which consists of $N_{q}$ outgoing and $N_{q}$ incoming waves (so that these also form bases of the respective subspaces $\mathbf{W}^{+}(\lambda)$ and $\left.\mathbf{W}^{-}(\lambda)\right)$.

Let $\lambda \in\left[\lambda_{q-1}^{\dagger}, \lambda_{q}^{\dagger}\right)$ with the notation of Section 2.2. In the case the eigenvalue $\mu_{j}(\lambda)$ in (2.12) is negative, we use the power-law solutions (2.18) to define

$$
w_{j}^{ \pm}(x)=\chi(x) r^{-1 \pm i\left|\mu_{j}(\lambda)\right|^{1 / 2}} V_{j}(\vartheta) .
$$

If $\mu_{j}(\lambda)=0$, we have according to (2.19), (2.20),

$$
w_{j}^{0}(x)=\chi(x) r^{-\lambda} V_{j}(\vartheta), w_{j}^{1}(x)=\chi(x) r^{-1} \ln r V_{j}(\vartheta) .
$$

The cut-off function $\chi$ with the support in the blanket is taken from (2.46).

The functions in (4.1), (4.2) are representatives of the equivalence classes in the quotient space $\mathbf{W}(\lambda)$. Since they are linearly independent and their number is exactly $2 N_{q}=\operatorname{dim} \mathbf{W}(\lambda)$, they form a basis in $\mathbf{W}(\lambda)$. However, we shall select another specific basis in order to establish the radiation conditions as $x \rightarrow \mathcal{O}$ in $\Xi_{R}$, and following [34], [35], (Chap. 5), we classify the waves by means of the symplectic form

$$
\mathbf{Q}_{\lambda}(\mathbf{w}, \mathbf{v})=\left(\mathbf{A}_{\beta}(\lambda) \mathbf{w}, \mathbf{v}\right)_{\Omega}-\left(\mathbf{w}, \mathbf{A}_{\beta}(\lambda) \mathbf{v}\right)_{\Omega} .
$$

Clearly,

$$
\mathbf{Q}_{\lambda}(\mathbf{w}, \mathbf{v})=-\overline{\mathbf{Q}_{\lambda}(\mathbf{v}, \mathbf{w})} .
$$


Since $\mathbf{A}_{\beta}(\lambda)$ is the restriction of $A_{\beta}(\lambda)=A_{-\beta}(\lambda)^{*}$ onto $\mathbf{V}_{\beta}(\lambda)$, we have in the cases $\mathbf{w} \in V_{-\beta}^{1}(\Omega)$ or $\mathbf{v} \in V_{-\beta}^{1}(\Omega)$

$$
\mathbf{Q}_{\lambda}(\mathbf{w}, \mathbf{v})=0 .
$$

In other words, the form (4.3) is actually defined on $\mathbf{W}(\lambda) \times \mathbf{W}(\lambda)$.

\subsection{Calculating the form}

From the definition of the operator $A_{\beta}(\lambda)$ in Section 1.2 and the Green formula, for $\varphi \in V_{\beta+1}^{2}(\Omega) \subset V_{\beta}^{1}(\Omega)$ and $\varphi \in V_{-\beta}^{1}(\Omega)$ we obtain

$$
\left(A_{\beta}(\lambda) \varphi, \psi\right)_{\Omega}=\left(-\Delta_{x} \varphi, \psi\right)_{\Omega}+\left(\partial_{z} \varphi-\lambda \varphi, \psi\right)_{\Gamma}+\left(\partial_{n} \varphi, \psi\right)_{\partial \Theta} .
$$

Let $\psi$ be a function in $y$ with support in $\bar{\Xi}_{R} \backslash \mathcal{O}$, i.e. $\psi \in C_{c}^{\infty}\left(\mathbb{B}_{R}^{\bullet}\right)$. We substitute $v_{j}^{\alpha}$ (see (4.1), (4.2)) for $\varphi$ and derive the identity

$$
\begin{aligned}
\left(\mathbf{A}_{\beta}(\lambda) v_{j}^{\alpha}, \psi\right)_{\Omega} & =\left(-h \Delta_{y} v_{j}^{\alpha}, \psi\right)_{\mathbb{B}_{R}}-\lambda\left(v_{j}^{\alpha}, \psi\right)_{\mathbb{B}_{R}}-\left(\nabla_{y} h \cdot \nabla_{y} v_{j}^{\alpha}, \psi\right)_{\mathbb{B}_{R}} \\
& =-\left(\nabla_{y} \cdot h \nabla_{y} v_{j}^{\alpha}-\lambda v_{j}^{\alpha}, \psi\right)_{\mathbb{B}_{R}} .
\end{aligned}
$$

Note that this calculation is quite similar to (3.18); the first factor $h$ comes from the integration in $z \in(-h(y), 0)$ and the term with $\nabla_{y} h$. is due to the formulas

$$
\begin{aligned}
\partial_{\nu} & =\left(1+\left|\nabla_{y} h(y)\right|^{2}\right)^{-1 / 2}\left(-\nabla_{y} h(y),-\partial_{z}\right), \\
d s_{x} & =\left(1+\left|\nabla_{y} h(y)\right|^{2}\right)^{1 / 2} d y \text { for } x \in \partial \Theta \cap \bar{\Xi}_{R} .
\end{aligned}
$$

In the vicinity of the point $y=0$ the function $v_{j}^{\alpha}$ constitutes a power-law solution of the limit equation (2.7), and by (1.3) we have $\left|\nabla_{y} \cdot h(y) \nabla_{y} v_{j}^{\alpha}(y)+\lambda v_{j}^{\alpha}(y)\right|=\mathcal{O}\left(r^{0}\right)$ as $r=|y| \rightarrow 0$ and

$$
\nabla_{y} \cdot h \nabla_{y} v_{j}^{\alpha}+\lambda v_{j}^{\alpha} \in L_{\varepsilon-1}^{2}\left(\mathbb{B}_{R}\right) \text { for any } \varepsilon>0 .
$$

Hence,

$$
\left|\left(\mathbf{A}_{\beta}(\lambda) v_{j}^{\alpha}, \psi\right)\right| \leq c\left\|\psi ; L_{1-\varepsilon}^{2}\left(\mathbb{B}_{R}\right)\right\| .
$$

Since $v_{p}^{\sigma} \in V_{1+\varepsilon}^{1}\left(\mathbb{B}_{R}\right) \subset L_{\varepsilon}^{2}\left(\mathbb{B}_{R}\right)$ can be approximated by functions in $C_{c}^{\infty}\left(\left(\overline{\mathbb{B}}_{R}\right) \bullet\right)$ in the $V_{1+\varepsilon}^{1}$-norm, $\varepsilon \in(0,1 / 2]$, we may replace $\psi$ by $v_{p}^{\sigma}$. As a result, we obtain that

$$
\mathbf{Q}_{\lambda}\left(v_{j}^{\alpha}, v_{p}^{\sigma}\right)=-\left(\nabla_{y} \cdot h \nabla_{y} v_{j}^{\alpha}+\lambda v_{j}^{\alpha}, v_{p}^{\sigma}\right)_{\mathbb{B}_{R}}+\left(v_{j}^{\alpha}, \nabla_{y} \cdot h \nabla_{y} v_{p}^{\sigma}+\lambda v_{p}^{\sigma}\right)_{\mathbb{B}_{R}} .
$$

We employ the Green formula and obtain

$$
\begin{aligned}
\mathbf{Q}_{\lambda}\left(v_{j}^{\alpha}, v_{p}^{\sigma}\right)= & \lim _{\varrho \rightarrow 0}\left(-\left(\nabla_{y} \cdot h \nabla_{y} v_{j}^{\alpha}+\lambda v_{j}^{\alpha}, v_{p}^{\sigma}\right)_{\mathbb{B}_{R} \backslash \mathbb{B}_{\varrho}}\right. \\
& \left.+\left(v_{j}^{\alpha}, \nabla_{y} \cdot h \nabla_{y} v_{p}^{\sigma}+\lambda v_{p}^{\sigma}\right)_{\mathbb{B}_{R} \backslash \mathbb{B}_{\varrho}}\right) \\
= & \lim _{\varrho \rightarrow 0} \int_{\mathbb{S}_{\varrho}^{1}} h(y)\left(\overline{v_{p}^{\sigma}(y)} \partial_{r} v_{j}^{\alpha}(y)-v_{j}^{\alpha}(y) \partial_{r} \overline{v_{p}^{\sigma}(y)}\right) \mathrm{d} s_{y} .
\end{aligned}
$$


Because of (1.3) the change $h \mapsto H$ does not influence the limit; this thus equals

$$
C_{j, p}^{\alpha, \sigma}(\ln \varrho) \varrho^{2-1+\mathrm{i} \alpha\left|\mu_{j}(\lambda)\right|^{1 / 2}-1-\mathrm{i} \sigma\left|\mu_{p}(\lambda)\right|^{1 / 2}},
$$

where $C_{j, p}^{\alpha, \sigma}(\ln \varrho)$ is at most a quadratic polynomial in $\ln \varrho(c f .(4.1),(4.2))$. The left-hand side of (4.8) is finite, and therefore the limit vanishes provided

$$
\alpha\left|\mu_{j}(\lambda)\right| \neq \sigma\left|\mu_{p}(\lambda)\right|
$$

moreover, the polynomial is constant.

Continuing our calculation, we consider the functions (4.1) and obtain using (2.16)

$$
\begin{aligned}
\mathbf{Q}_{\lambda}\left(v_{j}^{\alpha}, v_{p}^{\sigma}\right) & =\left(\left(-1+\mathrm{i} \alpha\left|\mu_{j}(\lambda)\right|^{1 / 2}\right)-\left(-1-\mathrm{i} \sigma\left|\mu_{p}(\lambda)\right|^{1 / 2}\right)\right)\left(\mathcal{H} V_{j}, V_{p}\right)_{\mathbb{S}^{1}} \\
& =2 \mathrm{i} \alpha\left|\mu_{j}(\lambda)\right|^{1 / 2} \delta_{j, p} \delta_{\alpha, \sigma} .
\end{aligned}
$$

Dealing with functions (4.2), we write

$$
\begin{aligned}
\mathbf{Q}_{\lambda}\left(\chi r^{-1}\left(a_{j}+b_{j} \ln r\right) V_{j}, \chi r^{-1}\left(a_{p}+b_{p} \ln r\right) V_{p}\right)= & \lim _{\varrho \rightarrow 0}\left(\left(\bar{a}_{p}+\bar{b}_{p} \ln \varrho\right)\left(b_{j}-a_{j}-b_{j} \ln \varrho\right)\right. \\
& \left.-\left(a_{j}+b_{j} \ln \varrho\right)\left(\bar{b}_{p}-\bar{a}_{p}-\bar{b}_{p} \ln \varrho\right)\right)\left(\mathcal{H} V_{j}, V_{p}\right)_{\mathbb{S}^{1}} \\
= & \left(\bar{a}_{p}\left(b_{j}-a_{j}\right)-a_{j}\left(\bar{b}_{p}-\bar{a}_{p}\right)\right) \delta_{j, p}=\left(\bar{a}_{p} b_{j}-a_{j} \bar{b}_{p}\right) \delta_{j, p} .
\end{aligned}
$$

Motivated by (4.9) and (4.10) we set

$$
\mathbf{w}_{j}^{ \pm}(x)=\frac{1}{\sqrt{2}}\left|\mu_{j}(\lambda)\right|^{-1 / 4} \chi(x) r^{-1 \pm \mathrm{i}\left|\mu_{j}(\lambda)\right|^{1 / 2}} V_{j}(\vartheta)
$$

in the case $\mu_{j}(\lambda)<0$, and, for $\mu_{j}(\lambda)=0$,

$$
\mathbf{w}_{j}^{ \pm}(x)=\frac{1}{\sqrt{2}} \chi(x) r^{-1 / 2}(1 \pm \mathrm{i} \ln r) V_{j}(\vartheta) .
$$

The waves (4.11) and (4.12) form the desired basis in $\mathbf{W}(\lambda)$ and they satisfy the relations

$$
\mathbf{Q}_{\lambda}\left(\mathbf{w}_{j}^{ \pm}, \mathbf{w}_{p}^{ \pm}\right)= \pm \mathrm{i} \delta_{j, p}, \mathbf{Q}_{\lambda}\left(\mathbf{w}_{j}^{ \pm}, \mathbf{w}_{p}^{\mp}\right)=0, j, p=1, \ldots, N_{q} .
$$

The waves with the plus sign are called outgoing to the point $\mathcal{O}$, while those with the minus sign are incoming from the point $\mathcal{O}$; the reason is explained in Section 4.5.

\subsection{The scattering matrix}

Since ker $\mathbf{A}_{\beta}(\lambda)=\operatorname{ker} A_{\beta}(\lambda)$, we decompose

$$
\operatorname{ker} \mathbf{A}_{\beta}(\lambda)=\operatorname{ker} A_{-\beta}(\lambda) \oplus \mathbf{K}(\lambda) .
$$

An element in $\operatorname{ker} A_{-\beta}(\lambda)$ is a solution in $H^{1}(\Omega)$ of the homogeneous problem (1.9) (or (1.6-1.8) in the differential form). These solutions are nothing but eigenfunctions of the problem (1.6-1.8) in $H^{1}(\Omega)$, corresponding to the eigenvalue $\lambda$. Analogously to $[10,42]$ and others, we call such solutions trapped modes. By (1.16), (1.17) 
and (3.33), the dimension of the subspace $\mathbf{K}(\lambda) \subset \mathbf{W}(\lambda)$ is equal to $N_{q}$. Let us show that $\mathbf{K}(\lambda)$ has a basis $\mathbf{y}_{1}, \ldots, \mathbf{y}_{N_{q}}$ such that

$$
\mathbf{y}_{j} \cong \mathbf{w}_{j}^{-}+\sum_{k=1}^{N_{q}} \mathbf{s}_{j k} \mathbf{w}_{j}^{+} \quad\left(\bmod V_{-\beta}^{1}(\Omega)\right)
$$

for some numbers $\mathbf{s}_{j k}$. First, let $\mathbf{y} \in \mathbf{K}(\lambda)$ be arbitrary. Suppose first that

$$
\mathbf{y} \cong \sum_{k=1}^{N_{q}} \mathbf{a}_{k} \mathbf{w}_{j}^{+} \quad\left(\bmod V_{-\beta}^{1}(\Omega)\right) .
$$

holds for some numbers $\mathbf{a}_{j}$. Recalling (4.4), (4.13) we obtain

$$
0=\mathbf{Q}_{\lambda}(\mathbf{y}, \mathbf{y})=\sum_{j, k=1}^{N_{q}} \mathbf{a}_{k} \overline{\mathbf{a}}_{j} \mathbf{Q}\left(\mathbf{w}_{k}^{+}, \mathbf{w}_{j}^{+}\right)=\mathrm{i} \sum_{j=1}^{N_{q}}\left|\mathbf{a}_{j}\right|^{2} .
$$

Hence, the linear combination (4.16) vanishes. Now, $\mathbf{K}(\lambda)$ has a basis of $N_{q}$ functions of the form

$$
\sum_{k=1}^{N_{q}} \mathbf{b}_{j k}^{-} \mathbf{w}_{k}^{-}+\sum_{k=1}^{N_{q}} \mathbf{b}_{j k}^{+} \mathbf{w}_{k}^{+} \quad\left(\bmod V_{-\beta}^{1}(\Omega)\right)
$$

for some numbers $\mathbf{b}_{j k}^{-}, \mathbf{b}_{j k}^{+}$. We deduce that the $N_{q}$ functions

$$
\sum_{k=1}^{N_{q}} \mathbf{b}_{j k}^{-} \mathbf{w}_{k}^{-} \quad\left(\bmod V_{-\beta}^{1}(\Omega)\right)
$$

form a linearly independent set. (The linear dependence of these functions would imply the linear dependence of the functions (4.18), by the calculation (4.16-4.17).) Applying the Gram-Schmidt method to the coefficient matrix $\left(\mathbf{b}_{j k}^{-}\right)$makes the basis (4.18) into the basis (4.15) of $\mathbf{K}(\lambda)$.

Theorem 4.1. In the subspace $\mathbf{K}(\lambda) \subset \operatorname{ker} \mathbf{A}_{\beta}(\lambda)$ one can find a basis $\mathbf{y}_{1}, \ldots, \mathbf{y}_{N_{q}}$ such that the decompositions (4.15) hold true. The coefficients $\mathbf{s}_{j k}$ form a unitary $\left(N_{q} \times N_{q}\right)$-matrix $\mathbf{s}$.

Proof. It suffices to establish the equality

$$
\mathbf{s}^{*}=\mathbf{s}^{-1}
$$

Similarly to (4.17) we obtain

$$
\begin{aligned}
\mathbf{Q}_{\lambda}\left(\mathbf{y}_{j}, \mathbf{y}_{k}\right) & =\mathbf{Q}_{\lambda}\left(\mathbf{w}_{j}^{-}+\sum_{p=1}^{N_{q}} \mathbf{s}_{j p} \mathbf{w}_{p}^{+}, \mathbf{w}_{k}^{-}+\sum_{m=1}^{N_{q}} \mathbf{s}_{k m} \mathbf{w}_{m}^{+}\right) \\
& =\mathbf{Q}_{\lambda}\left(\mathbf{w}_{j}^{-}, \mathbf{w}_{k}^{-}\right)+\sum_{p, m=1}^{N_{q}} \mathbf{s}_{j p} \overline{\mathbf{s}_{k m}} \mathbf{Q}_{\lambda}\left(\mathbf{w}_{p}^{+}, \mathbf{w}_{m}^{+}\right) \\
& =-\mathrm{i} \delta_{j k}+\mathrm{i} \sum_{p, m=1}^{N_{q}} \mathbf{s}_{j p} \overline{\mathbf{s}_{k m}} \delta_{p, m}=\mathrm{i}\left(\sum_{p, m=1}^{N_{q}} \mathbf{s}_{j p} \overline{\mathbf{s}_{k m}}-\delta_{j k}\right) .
\end{aligned}
$$

Since $\mathbf{A}_{\beta}(\lambda) \mathbf{y}_{p}=0$, this expression vanishes and the equality (4.19) is true. 


\subsection{Radiation conditions}

The space of waves admits the decomposition

$$
\mathbf{W}(\lambda)=\mathbf{W}^{+}(\lambda) \oplus \mathbf{W}^{-}(\lambda)
$$

where $\mathbf{W}^{ \pm}(\lambda)$ is the linear hull of the waves $\mathbf{w}_{1}^{ \pm}, \ldots, \mathbf{w}_{N_{q}}^{ \pm}$. We furthermore introduce the subspace

$$
\mathbf{V}_{\beta}^{+}(\lambda)=V_{-\beta}^{1}(\Omega) \oplus \mathbf{W}^{+}(\lambda)=\mathbf{V}_{\beta}(\lambda) \ominus \mathbf{W}^{-} \subset V_{\beta}^{1}(\Omega)
$$

and the restriction

$$
\mathbf{A}_{\beta}^{+}(\lambda): \mathbf{V}_{\beta}^{+}(\lambda) \rightarrow V_{\beta}^{1}(\Omega)^{*}
$$

of the operator $A_{\beta}(\Omega)$.

Theorem 4.2. The operator $\mathbf{A}_{\beta}^{+}(\lambda)$ in (4.22) is Fredholm of index zero. Moreover,

$$
\operatorname{ker} \mathbf{A}_{\beta}^{+}(\lambda)=\operatorname{ker} A_{-\beta}(\lambda), \quad \operatorname{coker} \mathbf{A}_{\beta}^{+}(\lambda)=\operatorname{coker} A_{\beta}(\lambda)=\operatorname{ker} A_{-\beta}(\lambda) .
$$

Proof. By (3.32) and (3.33) we have

$$
\operatorname{Ind} \mathbf{A}_{\beta}^{+}(\lambda)=\operatorname{Ind} \mathbf{A}_{\beta}(\lambda)-N_{q}=0 \text {. }
$$

Theorem 4.1 ensures the first equality in (4.23). Since $\mathbf{A}_{\beta}^{+}(\lambda)=\left.A_{\beta}(\lambda)\right|_{\mathbf{V}_{\beta}^{+}(\lambda)}$, we have coker $\mathbf{A}_{\beta}^{+}(\lambda) \supset$ coker $A_{\beta}(\lambda)$, and observing (4.24) we conclude with the second formula in (4.23).

The radiation conditions hidden in formulas (4.21), (4.22) ought to be interpreted in the framework of function spaces with weighted norms and separated asymptotics $\left(c f\right.$. $[28,29,33]$ and others). Indeed, the space $\mathbf{V}_{\beta}^{+}(\lambda)$ consists of functions of the form

$$
\varphi(x)=\widetilde{\varphi}(x)+\sum_{j=1}^{N_{q}} a_{j} \mathbf{w}_{j}^{+}(x)
$$

and has the norm, $c f .(3.31)$,

$$
\left\|\varphi ; \mathbf{V}_{\beta}^{+}(\lambda)\right\|=\left(\left\|\widetilde{\varphi} ; V_{-\beta}^{1}(\Omega)\right\|^{2}+\sum_{j=1}^{N_{q}}\left|a_{j}\right|^{2}\right)^{1 / 2}
$$

Traditionally the asymptotic form with unknown coefficients $a_{j}$ and the decaying remainder $\widetilde{\varphi}$ is regarded as radiation conditions. Theorem 4.2 tells us that, for any $F \in V_{\beta}^{1}(\Omega)^{*}$ satisfying the $J(\lambda)$ compatibility conditions (1.23) and with the basis $\varphi_{1}, \ldots, \varphi_{J(\lambda)}$, the problem (1.14) has a solution in the form (4.25). This solution is defined up to a linear combination of the eigenfunctions $\varphi_{1}, \ldots, \varphi_{J(\lambda)}$ (trapped modes), and under the orthogonality conditions (1.24) it admits the estimate

$$
\left\|\varphi ; \mathbf{V}_{\beta}^{-}(\lambda)\right\| \leq c(\lambda)\left\|F ; V_{\beta}^{1}(\Omega)^{*}\right\| .
$$

One readily observes that the above-mentioned facts on the solvability of the linear water-wave problem are exactly the same as for the Fredholm operator $A_{0}(\lambda)$ in the case $\lambda<\lambda_{\dagger}$ (see the text around (1.22-1.25)). 


\subsection{Discussion}

Theorem 4.2 provides a rigorous formulation of the radiation condition to describe the wave process inside the finite water blanket (1.5). Let us give a physical interpretation of the asymptotic representation (4.25). First, we assume that $\lambda>\lambda_{\dagger}$ and $\lambda \neq \lambda_{j}^{+}, j \in \mathbb{N}$.

Recall the formula $\lambda=\omega^{2} / g$ for the spectral parameter $\lambda$ and the oscillating factor $\exp (\mathrm{i} \omega t)$, which comes from the time-dependent boundary condition

$$
\frac{\partial^{2} \varphi}{\partial t^{2}}(t, y, 0)+g \frac{\partial \varphi}{\partial z}(t, y, 0)=0, x \in \Gamma
$$

for the equilibrium of the water surface (see, e.g. [15,41]). Based on this, (4.1) and (4.11) we write

$$
r \exp (\mathrm{i} \omega t) \mathbf{w}_{j}^{ \pm}(x)=\frac{1}{\sqrt{2}} \chi(x)\left|\mu_{j}(\lambda)\right|^{-1 / 2} \exp \left(\mathrm{i}\left(\omega t \pm\left|\mu_{j}(\lambda)\right|^{1 / 2} \ln r\right)\right) V_{j}(\vartheta) .
$$

Ignoring the first factor $r$ on the left (see an explanation below), we see that the right-hand side of (4.28) stays unchanged, if the $t$ - and $r$-variables are related by

$$
t=\mp \omega^{-1}\left|\mu_{j}(\lambda)\right|^{1 / 2} \ln r \Leftrightarrow r=\exp \left(\mp\left|\mu_{j}(\lambda)\right|^{-1 / 2} \omega t\right) .
$$

We then observe that in the plus case in (4.28), increasing $t$ leads to diminishing $r$ in (4.29). This means that the wave $\mathbf{w}_{j}^{+}$propagates towards the point $\mathcal{O}$. The minus case in contrast corresponds to a wave which propagates from $\mathcal{O}$ inside the blanket to the water massive.

The radiation conditions (4.25) only allow waves propagating towards the point $\mathcal{O}$. By (4.29), at a fixed time $t^{\prime}$ the wave (4.28) appears at the distance

$$
r^{\prime}=\exp \left(-\omega\left|\mu_{j}(\lambda)\right|^{-1 / 2} t^{\prime}\right)
$$

from $\mathcal{O}$, and this tends to 0 , if $t^{\prime} \rightarrow \infty$. Thus, it takes an infinite time for this wave to reach the point, which in this sense sucks the outgoing waves and becomes "a black hole" for water-waves; in other words, a wave entering the blanket never leaves it. For analogous effects in acoustics and elasticity, cf. [5,14,23] and others.

In analogy with diffraction in cylindrical domains, we recognize $\mp\left|\mu_{j}(\lambda)\right|^{1 / 2} e_{(r)}$ as the wave vector of $\mathbf{w}_{j}^{ \pm}$; here $e_{(r)}=(\cos \vartheta, \sin \vartheta, 0)$. The radiation conditions $(4.25)$ select waves whose vectors are directed to $\mathcal{O}$ and are therefore of Sommerfeld's type. We consider below a radiation principle of Mandelstam's type, concerning the energy flux. We emphasize that none of these physical radiation principles applies to standing $\left(\mu_{j}(\lambda)=0\right)$ waves. However, our radiation conditions (4.25) become physical in the nonthreshold $\left(\lambda \neq \lambda_{\dagger}\right)$ situation.

According to the Mandelstam principle the direction of a propagating wave $\mathbf{W}$ is determined by the sign of the integral

$$
\int_{\xi_{\rho}} e_{(r)} \cdot \widehat{I}(\mathbf{w}) \mathrm{d} s_{x}
$$

where $\widehat{I}(\mathbf{w})$ is the mean value of the Poynting vector $I(\mathbf{w})$ over $(0,2 \pi / \omega) \ni t$. The integral (4.30) yields the energy flux through the surface $\xi_{\rho}=\left\{x \in \Xi_{R}: r=\rho\right\}$; here $\rho<R$. To determine the Poynting vector, we consider a domain $G$ inside $\Omega$ with a smooth surface $\partial G$. The rate of the change of the energy kept inside $G$ is defined by

$$
J:=\frac{\partial}{\partial t} \int_{G}\left|\nabla_{x} \operatorname{Re} \mathbf{E w}\right|^{2} \mathrm{~d} x=\int_{\partial G} \nu \cdot I(\mathbf{w}) \mathrm{d} s_{x},
$$


where $\mathbf{E w}=\exp (-\mathrm{i} \omega t) \mathbf{w}$ and the integral on the right of (4.31) involves the vector function $I(\mathbf{w})$ to be determined. We have

$$
J=2 \omega \int_{G} \nabla_{x}(\operatorname{Re} \mathbf{E w}) \cdot \nabla_{x}(\operatorname{Im} \mathbf{E w}) \mathrm{d} x=2 \omega \int_{\partial G} \operatorname{Re}(\mathbf{E w}) \nu \cdot \nabla_{x}(\operatorname{Im} \mathbf{E w}) \mathrm{d} s_{x}
$$

so that $I(\mathbf{w})=2 \omega(\operatorname{Re} \mathbf{E w}) \nabla_{x}(\operatorname{Im} \mathbf{E w})$ and

$$
\begin{aligned}
\widehat{I}(\mathbf{w}) & =\frac{\omega^{2}}{\pi} \int_{0}^{2 \pi / \omega} I(\mathbf{w}) \mathrm{d} t=-\frac{\omega^{2}}{4 \pi} \int_{0}^{2 \pi / \omega}(\mathbf{E w}+\overline{\mathbf{E w}}) \nabla_{x}(\mathbf{E w}-\overline{\mathbf{E w}}) \mathrm{d} t \\
& =\frac{\omega^{2}}{4 \pi} \int_{0}^{2 \pi / \omega}\left(\mathbf{w} \nabla_{x} \overline{\mathbf{w}}-\overline{\mathbf{w}} \nabla_{x} \mathbf{w}\right) \mathrm{d} t=\frac{\omega}{2}\left(\mathbf{w} \nabla_{x} \overline{\mathbf{w}}-\overline{\mathbf{w}} \nabla_{x} \mathbf{w}\right) .
\end{aligned}
$$

We now conclude that the integral (4.30) is equal to

$$
\frac{\omega}{2} \int_{\xi_{p}}\left(\mathbf{w} \partial_{r} \overline{\mathbf{w}}-\overline{\mathbf{w}} \partial_{r} \mathbf{w}\right) \mathrm{d} s_{x}=\frac{\omega}{2} \int_{\mathbb{S}_{\rho}^{1}} h\left(\mathbf{w} \partial_{r} \overline{\mathbf{w}}-\overline{\mathbf{w}} \partial_{r} \mathbf{w}\right) \mathrm{d} s_{y} .
$$

Owing to the previous calculations in Section 4.2, the limit $\rho \rightarrow 0$ of the expression (4.32) is simply proportional to the symplectic form $\mathbf{Q}(\mathbf{w}, \mathbf{w})$. Thus, the Mandelstam principle leads to our radiation conditions (4.25) as well.

We emphasize that the above calculation is not true for the power-logarithmic solution (2.20) (see also (4.2) and (4.12)). We directly extend the division (4.20) of waves according to the symplectic form (4.3) which is mathematically natural and therefore the radiation conditions (4.25) are called intrinsic in the threshold situation.

\section{A. Appendix: The spectrum of the Self-Adjoint operator of the linear WATER-WAVE PROBLEM}

We complete the paper by a study of the spectrum of (1.6-1.8). Following the approach of [38], we introduce the Hilbert space (called Maz'ya space) with norm

$$
\|u ; \mathcal{H}\|=\left(\left\|u ; H^{1}(\Omega)\right\|^{2}+\left\|u ; L^{2}(\Gamma)\right\|^{2}\right)^{1 / 2}
$$

and scalar product

$$
\langle\varphi, \psi\rangle=\left(\nabla_{x} \varphi, \nabla_{x} \psi\right)_{\Omega}+(\varphi, \psi)_{\Gamma} .
$$

Let the operator $\mathcal{T}$ in $\mathcal{H}$ given by

$$
\langle\mathcal{T} \varphi, \psi\rangle=(\varphi, \psi)_{\Gamma}, \varphi, \psi \in \mathcal{H} ;
$$

it is evidently continuous and symmetric, therefore, self-adjoint. Moreover, it is positive and $\|\mathcal{T} ; \mathcal{H}\| \leq 1$, thus, the spectrum of $\mathcal{T}$ belongs to the closed segment $[0,1]$. Besides, the point $\tau=0$ is an eigenvalue of $\mathcal{T}$ with infinite multiplicity and eigenspace

$$
\left\{\varphi \in H^{1}(\Omega): \varphi=0 \text { on } \Gamma\right\} .
$$


As shown in [38], the embedding $H^{1}(\Omega) \subset L^{2}(\Gamma)$ is not compact and, hence, $\tau=0$ cannot be the only point of the essential spectrum of $\mathcal{T}$ (see, e.g., [2], Thm. 9.2.1). We prove the following assertion and present a description of the upper bound $\tau_{\dagger} \in(0,1)$ of the essential spectrum.

Theorem A.1. The essential spectrum of the operator $\mathcal{T}$ is $\left[0, \tau_{\dagger}\right]$, where $\tau_{\dagger}=\left(1+\lambda_{\dagger}\right)^{-1} \in(0,1)$. The half-open segment $\left(0, \tau_{\dagger}\right]$ is covered by the continuous spectrum of $\mathcal{T}$.

Recall that $\lambda_{\dagger}$ was defined in Section 2.2, and it depends only on the coefficients $H_{p q}$ in (1.4).

Before proceeding with the proof we remark that the problem (1.9) with $f=0$ is equivalent to the abstract equation

$$
\mathcal{T} \varphi=\tau \varphi \text { in } \mathcal{H}
$$

with the new spectral parameter

$$
\tau=(1+\lambda)^{-1}
$$

To derive (A.4) is just to add $(\varphi, \psi)_{\Gamma}$ to both sides of (1.9) and to take definitions (A.2), (A.3) into account. The relation (A.5) between the spectral parameters passes all properties of the spectrum of $\mathcal{T}$ to the spectrum of (1.6)-(1.8); the only exception is the point $\tau=0$ which is transformed to infinity. The claim of Theorem A.1 on the continuous spectrum can be rephrased so that the continuous spectrum of the water-wave problem in $\Omega$ coincides with the ray $\left[\lambda_{\dagger},+\infty\right)$ where

$$
\lambda_{\dagger}=\tau_{\dagger}^{-1}-1 .
$$

Below the threshold (A.6) the problem has discrete spectrum. In particular, $\lambda=0$ is a simple eigenvalue with the eigenfunction $\varphi=$ const.

Proof of Theorem A.1. The inequality

$$
\left\|\varrho^{-1} u ; L^{2}(\Omega)\right\| \leq c\left\|u ; H^{1}(\Omega)\right\|
$$

was proven in [38]. Hence, in view of Lemma 1.1 and the definition (1.10) with $\beta=0$, the Hilbert space $\mathcal{H}$ with the scalar product (A.2) coincides algebraically and topologically with the weighted space $V_{0}^{1}(\Omega)$ so that

$$
\|u ; \mathcal{H}\| \leq c\left\|u ; V_{0}^{1}(\Omega)\right\| \leq C\|u ; \mathcal{H}\| .
$$

Furthermore, according to the definition (A.3) of the operator $\mathcal{T}$ in $\mathcal{H}$, the equation

$$
\mathcal{T} \varphi-\tau \varphi=\mathcal{F} \text { in } \mathcal{H}
$$

with $\tau \in(0,1]$ and $\mathcal{F} \in \mathcal{H}$, is equivalent to the variational problem (1.14), where $\beta=0, \lambda=\tau^{-1}-1$ and $F(\varphi)=-\tau^{-1}\langle\mathcal{F}, \varphi\rangle, F \in V_{0}^{1}(\Omega)^{*}$. This observation implies that in the case the operator $A_{0}(\lambda)$ is Fredholm, the point $\tau=-(1+\lambda)^{-1}$ falls either into the resolvent set, or into the discrete spectrum of $\mathcal{T}$.

If $\tau \in\left(0, \tau_{+}\right]$, then $\lambda=\tau^{-1}-1 \geq \lambda_{+}$, and therefore the operator $A_{0}(\lambda)$ is no longer Fredholm, by Theorem 3.2. This property is inherited by $\tau$, and $\lambda$ thus falls into the continuous spectrum. It suffices to recall that the kernel of $A_{0}(\lambda)$ is finite dimensional (see (3.9)) and the same holds true for $\operatorname{ker}(\mathcal{T}-\tau)$, hence, the interval $\left(0, \tau_{+}\right]$ is included in the continuous spectrum.

Acknowledgements. The authors thank the referees for careful reading of the manuscript and many suggestions which were helpful to improve the presentation of the paper. 


\section{REFERENCES}

[1] F.L. Bakharev and S.A. Nazarov, On the structure of the spectrum of the elasticity problem for a body with a super-sharp spike. Sibirsk. Mat. Zh. 50 (2009) 746-756. (English transl. Siberian Math. J. 50 (2009).)

[2] M.S. Birman and M.Z. Solomyak, Spectral theory of self-adjoint operators in Hilber space. Reidel Publ. Company, Dordrecht (1986).

[3] A.-S. Bonnet-Ben Dhia, P. Joly, Mathematical analysis of guided water waves. SIAM J. Appl. Math. 53 (1993).

[4] G. Cardone, S.A. Nazarov and J. Sokolowski, Asymptotics of solutions of the Neumann problem in a domain with closely posed components of the boundary. Asymptotic Analysis 62 (2009) 41-88.

[5] G. Cardone, S.A. Nazarov and J. Taskinen, The "absorption" effect caused by beak-shaped boundary irregularity for elastic waves. Dokl. Ross. Akad. Nauk. 425 (2009) 182-186. (English transl. Doklady Physics 54 (2009) 146-150.)

[6] G. Cardone, S.A. Nazarov and J. Taskinen, Criteria for the existence of the essential spectrum for beak-shaped elastic bodies. J. Math. Pures Appl. (to appear)

[7] D. Daners, Robin boundary value problems on arbitrary domains. Trans. Amer. Math. Soc. 352 (2000) 4207-4236.

[8] D. Daners, A Faber-Krahn inequality for Robin problems in any space dimension. Math. Annal. 335 (2006) 767-785.

[9] D. Gilbarg and N. Trudinger, Elliptic partial differential equations of second order. Die Grundlehren der mathematischen Wissenschaften 224. Springer, Berlin (1977).

[10] D.S. Jones, The eigenvalues of $\nabla^{2} u+\lambda u=0$ when the boundary conditions are given on semi-infinite domains. Proc. Camb. Phil. Soc. 49 (1953) 668-684.

[11] V.A. Kondratiev, Boundary value problems for elliptic problems in domains with conical or corner points. Trudy Moskov. Mat. Obshch. 16 (1967) 209-292. (English transl. Trans. Moscow Mat. Soc. 16 (1967) 227-313.)

[12] V.A. Kozlov, V.G. Maz'ya and J. Rossmann, Elliptic boundary value problems in domains with point singularities. Mathematical Surveys and Monographs 52. American Mathematical Society, Providence, RI (1997).

[13] V.A. Kozlov, V.G. Maz'ya and J. Rossmann, Spectral problems associated with corner singularities of solutions to elliptic equations. Mathematical Surveys and Monographs 85. American Mathematical Society, Providence, RI (2001).

[14] V.V. Krylov, New type of vibration dampers utilising the effect of acoustic "black holes". Acta Acustica united with Acustica 90 (2004) 830-837.

[15] N. Kuznetsov, V. Maz'ya and B. Vainberg, Linear Water Waves. Cambridge University Press, Cambridge (2002).

[16] O.A. Ladyzhenskaya, Boundary value problems of mathematical physics. Springer Verlag, New York (1985).

[17] J.L. Lions and E. Magenes, Non-homogeneus boundary value problems and applications (French). Dunod, Paris (1968). (English transl. Springer-Verlag, Berlin-Heidelberg-New York (1972).)

[18] V. Mazya, Sobolev spaces, translated from the Russian by T.O. Shaposhnikova. Springer Series in Soviet Mathematics. SpringerVerlag, Berlin (1985).

[19] V.G. Maz'ya and B.A. Plamenevskii, The asymptotic behavior of solutions of differential equations in Hilbert space. (Russian) Izv. Akad. Nauk SSSR Ser. Mat. 36 (1972) 1080-1133; erratum, Izv. Akad. Nauk SSSR Ser. Mat. 37 (1973) 709-710.

[20] V.G, Mazja and B.A. Plamenevskii, On coefficients in asymptotics of solutions of elliptic boundary value problems in a domain with conical points. Math. Nachr. 76 (1977) 29-60. (Engl. transl. Amer. Math. Soc. Transl. 123 (1984) 57-89.)

[21] V.G. Mazja and B.A. Plamenevskii, Estimates in $L_{p}$ and Hölder classes and the Miranda-Agmon maximum principle for solutions of elliptic boundary value problems in domains with singular points on the boundary. Math. Nachr. 81 (1978) 25-82. (Engl. transl. Amer. Math. Soc. Transl. (Ser. 2) 123 (1984) 1-56 .)

[22] V.G. Mazya and S.V. Poborchi, Imbedding and Extension Theorems for Functions on Non-Lipschitz Domains. SPbGU publishing (2006).

[23] M.A. Mironov, Propagation of a flexural wave in a plate whose thickness decreases smoothly to zero in a finite interval. Soviet Physics-Acoustics 34 (1988) 318-319.

[24] S.A. Nazarov Asymptotics of the solution of the Neumann problem at a point of tangency of smooth components of the boundary of the domain. Izv. Ross. Akad. Nauk. Ser. Mat. 58 (1994) 92-120. (English transl. Math. Izvestiya 44 (1995) 91-118.)

[25] S.A. Nazarov, On the flow of water under a still stone. Mat. Sbornik 186 (1995) 75-110. (English transl. Math. Sbornik 186 (1995) 1621-1658.)

[26] S.A. Nazarov, A general scheme for averaging self-adjoint elliptic systems in multidimensional domains, including thin domains. Algebra Analiz. 7 (1995) 1-92. (English transl. St. Petersburg Math. J. 7 (1996) 681-748.)

[27] S.A. Nazarov, The polynomial property of self-adjoint elliptic boundary-value problems and the algebraic description of their attributes. Uspehi Mat. Nauk. 54 (1999) 77-142. (English transl. Russ. Math. Surveys. 54 (1999) 947-1014.)

[28] S.A. Nazarov, Weighted spaces with detached asymptotics in application to the Navier-Stokes equations. in: Advances in Mathematical Fluid Mechanics. Paseky, Czech. Republic (1999) 159-191. Springer-Verlag, Berlin (2000).

[29] S.A. Nazarov, The Navier-Stokes problem in a two-dimensional domain with angular outlets to infinity. Zap. Nauchn. Sem. St.-Petersburg Otdel. Mat. Inst. Steklov 257 (1999) 207-227. (English transl. J. Math. Sci. 108 (2002) 790-805.)

[30] S.A. Nazarov, The spectrum of the elasticity problem for a spiked body. Sibirsk. Mat. Zh. 49 (2008) 1105-1127. (English transl. Siberian Math. J. 49 (2008) 874-893.) 
[31] S.A. Nazarov, On the spectrum of the Steklov problem in peak-shaped domains. Trudy St.-Petersburg Mat. Obshch. 14 (2008) 103-168. (English transl. Am. Math. Soc. Transl Ser. 2.)

[32] S.A. Nazarov. On the essential spectrum of boundary value problems for systems of differential equations in a bounded peakshaped domain. Funkt. Anal. i Prilozhen. 43 (2009) 55-67. (English transl. Funct. Anal. Appl. 43 (2009).)

[33] S.A. Nazarov and K. Pileckas, On steady Stokes and Navier-Stokes problems with zero velocity at infinity in a three-dimensional exterior domain. Journal of Mathematics of Kyoto University 40 (2000) 475-49.

[34] S.A. Nazarov and B.A. Plamenevskii, Radiation principles for self-adjoint elliptic problems. Probl. Mat. Fiz. 13. $192-244$. Leningrad: Leningrad Univ. 1991 (Russian).

[35] S.A. Nazarov and B.A. Plamenevskii, Elliptic problems in domains with piecewise smooth boundaries. Walter be Gruyter, Berlin, New York (1994).

[36] S.A. Nazarov and O.R. Polyakova, Asymptotic behavior of the stress-strain state near a spatial singularity of the boundary of the beak tip type. Prikl. Mat. Mekh. 57 (1993) 130-149. (English transl. J. Appl. Math. Mech. 57 (1993) 887-902.)

[37] S.A. Nazarov and S.A. Taskinen, On the spectrum of the Steklov problem in a domain with a peak. Vestnik St. Petersburg Univ. Math. 41 (2008) 45-52.

[38] S.A. Nazarov and J. Taskinen, On essential and continuous spectra of the linearized water-wave problem in a finite pond. Math. Scand. 106 (2009) 1-20.

[39] J. Peetre, Another approach to elliptic boundary problems. Comm. Pure. Appl. Math. 14 (1961) 711-731.

[40] B.A. Plamenevskii, The asymptotic behavior of the solutions of quasielliptic differential equations with operator coefficients. (Russian) Izv. Akad. Nauk SSSR Ser. Mat. 37 (1973) 1332-1375.

[41] J.J. Stoker, Water waves. The Mathematical Theory with Applications. Reprint of the 1957 original. John Wiley, New York (1992).

[42] F. Ursell, Trapping modes in the theory of surface waves. Proc. Camb. Phil. Soc. 47 (1951) 347-358. 